Check for updates

Cite this: Anal. Methods, 2017, 9, 2768

\title{
Recent progress in the application of nanomaterials in the analysis of emerging chemical contaminants
}

\author{
Xiu Huang, ${ }^{\text {ab }}$ Qian Liu, (D)*bcd Shouzhuo Yao*a and Guibin Jiang ${ }^{\text {bd }}$
}

Emerging chemical contaminants (ECCs) represent newly identified chemical contaminants that are not yet covered by routine monitoring and regulatory programs. Current research into ECCs is greatly hindered by the shortage of analytical methods due to the complex sample matrices, extremely low environmental levels, and the "emerging" nature of ECCs. The use of nanomaterials (NMs) to solve the analytical problem of ECCs is a very active research field. We published an earlier review paper summarizing the application of nanomaterials in the analysis and monitoring of ECCs in 2014. In the subsequent years, this field has continued to rapidly develop with many new materials and technologies emerging. This paper therefore reviews recent progress in this field. Different types of NMs and analytical techniques are covered. Notably, we pay special attention to newly developed materials, such as metal-organic frameworks (MOFs), upconversion materials, carbon dots, and titania $\left(\mathrm{TiO}_{2}\right)$ nanoparticles. We also stress the progress in emerging techniques, such as surface-enhanced Raman spectroscopy (SERS) and laser desorption/ionization mass spectrometry (LDI-TOF MS). Finally, we discuss current challenges and future prospects in this field.

Received 31st March 2017

Accepted 13th April 2017

DOI: $10.1039 / c 7 a y 00859 \mathrm{~g}$

rsc.li/methods
(PPCPs), hormones, perfluorinated compounds (PFCs), flame retardants, industrial additives, disinfection by-products (DBPs), perchlorate, algal toxins, etc. ${ }^{4}$

To date, knowledge of the occurrence of ECCs in the environment is still limited. ${ }^{5-8}$ Reliable ecotoxicological data are available for only a small fraction of ECCs. A serious bottleneck in ECCs studies is the lack of proper analytical methods. The difficulties in the analysis of ECCs lie mainly in the complexity of the environmental matrices, the extremely low environmental levels of ECCs (usually at ppb to ppt levels), and their "emerging" nature. ${ }^{4}$ In spite of the great efforts made in the development of analytical methods for ECCs in recent years, challenges still remain for most ECCs. ${ }^{3,9}$ The current analysis of ECCs in environmental samples is predominantly based on liquid or gas chromatographic separations (LC or GC) and mass spectrometric (MS) detection. Prior to analysis, rigorous sample pretreatment procedures are required, which are, however, laborious and time-consuming. ${ }^{10}$ The low throughput of these methods also greatly limits their application in environmental health and exposomics studies. Thus, it is highly desirable to develop new methods for the analysis of ECCs.

Due to their unique physical and chemical properties, nanomaterials (NMs) have been widely used in various fields. ${ }^{11-13}$ The use of NMs in analytical methods also represents an increasing trend in environmental analysis. ${ }^{\mathbf{1 4 , 1 5}}$ Specifically, NMs have shown great potential to be used as promising tools to solve the analytical issue of ECCs. For example, in sample preparation, NMs can be used as nanoabsorbents to replace traditional adsorbents owing to their high surface area and high 
affinity for chemical contaminants. ${ }^{16,17}$ For electrochemical detection, NMs can be used as highly efficient electrode materials or catalysts due to their exceptional photoelectric properties and size effects. ${ }^{18,19}$ Apart from these, matrix-assisted laser desorption/ionization mass spectrometry (MALDI-TOF MS) is also an important application aspect of NMs. ${ }^{20}$ With strong optical absorption, excellent energy-transfer efficiency, and high chemical and physical stability, NMs can be used as novel MALDI matrices to extend the range of targeted analytes from biopolymers to small molecules. ${ }^{21-23}$ In 2014, we published a comprehensive review paper that summarized the applications of NMs in the analysis and monitoring of ECCs. ${ }^{4}$ In the last three years, we have noticed that this field has continued to undergo rapid development, especially in the following aspects:

(1) More NMs have gained new applications in the analysis of ECCs, e.g., metal-organic frameworks (MOFs), upconversion materials, carbon dots, and $\mathrm{TiO}_{2}$ nanoparticles;

(2) New technologies (e.g., surface-enhanced Raman spectroscopy (SERS) and MALDI-TOF MS) have been used more frequently in the analysis of ECCs;

(3) More types of ECCs have been analyzed by NMs-based analytical methods; and

(4) More NMs-based methods have been applied in studying real environmental systems.

In this article, we review recent progress in the application of NMs in the analysis of ECCs, especially the progress since 2014. We discuss the properties of several typical NMs used in environmental analysis and pay particular attention to the newly developed materials. We then summarize the applications of NMs in the determination of ECCs in terms of the different analytical techniques, such as sample preparation, electrochemical sensing, fluorescence detection, colorimetric detection, SERS, LDI-TOF MS, etc. It should be noted that we do not strictly limit our discussions to ECCs, and some representative methods toward traditional chemical contaminants are also included. We also discuss current challenges and give our perspectives in this field. Last but not least, we should keep in mind that NMs themselves are considered as a type of ECC, and the use of NMs should also be regulated and controlled within a safe extent. ${ }^{\mathbf{1 4 , 2 4 - 2 6}}$

\section{Nanomaterials used in environmental analysis}

\subsection{Common nanomaterials}

A great variety of NMs have gained different degrees of success in the analysis of ECCs. In our last review, ${ }^{4}$ we introduced in detail four kinds of common NMs, specifically carbon NMs, Au nanoparticles (Au NPs), magnetic nanoparticles (MNPs), and quantum dots (QDs). In recent applications, these materials have continued to play important roles in the analysis of ECCs. Some new carbon NMs have also emerged derived from their original forms by chemical modifications, such as oxidation, ${ }^{27,28}$ sulfonation, ${ }^{29-31}$ and fluorination. ${ }^{32-34}$ New analogs of carbon NMs, such as graphynes, ${ }^{35-40}$ graphene oxide nanoribbons (GONRs), ${ }^{27,41}$ ordered mesoporous carbon, ${ }^{42,43}$ and carbon nitride nanosheets, ${ }^{\mathbf{4 4 - 4 7}}$ have attracted increasing interest owing to their extraordinary physical and chemical properties. For ordered mesoporous carbon, its size-selective adsorption ability has been used to selectively enrich low-mass ECCs. ${ }^{48,49}$ For magnetic NMs, some novel materials have been prepared, such as carbon nitride nanosheets-supported silica-coated magnetic nanoparticles ${ }^{\mathbf{4 4 , 4 5 , 5 0}}$ and hybrid magnetic $\mathrm{CoFe}_{2} \mathrm{O}_{4}$ nanoparticles. ${ }^{51}$ For nonmagnetic NMs, titanium, copper, and zinc oxides have been prepared and applied in electrochemical sensing and in the SERS detection of ECCs in addition to the normally used Au and Ag nanoparticle-based substrates.

Besides the NMs above-mentioned, several novel types of NMs have also shown their tremendous application potentials in the analysis of ECCs in recent years, including MOFs, upconversion materials, carbon dots, and $\mathrm{TiO}_{2}$ nanoparticles. Herein we give a detailed introduction to these materials.

\subsection{Metal-organic frameworks}

MOFs have been applied in the analysis of ECCs due to their large surface areas, three-dimensional (3D) extended porous structure, excellent optical sensitivity, and structural and chemical tunability. ${ }^{52-56}$ MOFs are infinite crystalline lattices formed by the incorporation of metal ions into organic molecule ligands. The structure of MOFs is flexible and adjustable depending on the different metallic component vertices and organic linkers. The synthetic approaches of MOFs are rather diverse, among which the one-pot solvothermal method is most commonly used due to its ease of operation. ${ }^{53-55}$ Regarding environmental analysis, the poor stability in aqueous solution of MOFs is a great challenge. To overcome this problem, some water-stable MOFs have been synthesized, ${ }^{57}$ which can maintain as stable over relatively long periods of time over a wide $\mathrm{pH}$ range.

Besides MOFs, covalent-organic frameworks (COFs) are another emerging class of crystalline porous polymers with atomically precise designed and highly ordered organic building blocks. ${ }^{58-60}$ Similar to MOFs, COFs also have periodic and porous nanostructures and large surface areas, making COFs excellent candidates as sorbent materials or components in designing nanodevices for the analysis of ECCs.

\subsection{Upconversion nanomaterials}

Upconversion NMs, mainly consisting of lanthanide (Ln), yttrium (Y), and scandium (Sc) compounds, have recently attracted widespread attention. ${ }^{61}$ One of their most attractive properties is that they can emit light with shorter wavelengths than the excitation light. That is to say, it is possible to obtain visible light by near-infrared irradiation with upconversion NMs. ${ }^{\mathbf{6 1 , 6 2}}$ Therefore, this type of material is suitable for application in in situ and non-destructive biosensing, optical imaging, and photodynamic therapy. A key to the use of upconversion NMs is to overcome the limitation of low luminescence efficiency by choosing appropriate host materials. Halides (e.g., fluoride, chloride, and bromide) are used to effectively enhance upconversion luminescence efficiency. $\mathrm{Yb}^{3+}-\mathrm{Er}^{3+}$ or $\mathrm{Yb}^{3+}-\mathrm{Tm}^{3+}$ co-doped $\mathrm{NaYF}_{4}$ (e.g. , $\mathrm{NaYF}_{4}: \mathrm{Yb}^{3+}, \mathrm{Er}^{3+}$; $\mathrm{NaYF}_{4}: \mathrm{Yb}^{3+}, \mathrm{Tm}^{3+}$ ) are the most commonly used upconversion NMs owing to their high refractive index and high transparency. 
Different strategies have been developed for the synthesis of upconversion NMs, such as thermal decomposition, hydrothermal reaction, and ionic liquids-based synthesis. ${ }^{63}$ In the analysis of ECCs, the surface of upconversion NMs usually needs to be modified by functional groups, such as silica, polymers, molecularly imprinted polymers (MIPs), aptamers, proteins, DNA, biomacromolecules. ${ }^{61-63}$ These functionalizations can be achieved by ligand engineering, ligand attraction, surface polymerization, self-assembly, and layer-by-layer assembly technologies. ${ }^{61-63}$

\subsection{Carbon dots}

Carbon dots are a new type of carbon-based NMs. They have been shown to be suitable for bioimaging, disease diagnosis, catalysis, and photovoltaic devices owing to their chemical inertness, low toxicity, good biocompatibility, water solubility, high photostability, and low cost. ${ }^{\mathbf{6 4 , 6 5}}$ Carbon dots can be synthesized by topdown or bottom-up routes. The top-down approach is to break the large carbon structures to generate carbon dots. Carbon nanotubes (CNTs), graphite, activated carbon, and nanodiamonds can be used as candidate carbon sources. The bottomup approach requires a molecular precursor subjected to combustion/thermal treatments. Citrate, carbohydrates, and polymer-silica nanocomposites have been reported as available molecular precursors. ${ }^{\mathbf{6 , 6 7}}$ Nevertheless, naked carbon dots have low quantum yields because of their unstable surface defects, which reduce the ability of radiative recombination. Thus, surface passivation and functionalization are usually needed to reduce the harmful effects of carbon dots. Polymers, e.g., poly (propionyl ethyleneimine-co-ethyleneimine) (PPEI-EI), and $\mathrm{PEG}_{1500 \mathrm{~N}}$, are commonly used as surface passivating agents by forming insulating layers at the surface of the carbon dots. ${ }^{65}$

\section{5. $\mathrm{TiO}_{2}$ nanoparticles}

$\mathrm{TiO}_{2}$ nanoparticles can be used in different forms, i.e., powder, crystals, and thin films. $\mathrm{TiO}_{2}$ nanoparticles can be prepared by sol-gel, microemulsion, precipitation, hydrothermal, solvothermal, electrochemical, and biological strategies. ${ }^{68-70}$ The liquid-phase method is the most commonly used because of its convenience and ease of control. In the synthesis, it is essential to control the shape and size of the $\mathrm{TiO}_{2}$ nanoparticles by solgel or microemulsion processes. $\mathrm{TiO}_{2}$ nanoparticle have been widely used in photovoltaics, photocatalysis, photochromics, and sensors due to their excellent optical properties. ${ }^{\text {70,71 }}$ The applications have also been extended to the detection and removal of chemical contaminants.

\section{Recent applications of nanomaterials in the detection of emerging chemical contaminants}

\subsection{Sample preparation}

In addition to traditional solid-phase extraction (SPE) and solidphase microextraction (SPME) ${ }^{4}$ micro-solid-phase extraction $(\mu-$ $\mathrm{SPE})^{72,73}$ has recently sparked much research interest because of its low cost and capability for automation. Magnetic solid-phase extraction (MSPE) is another important technique in sample preparation. NMs provide promising tools to facilitate these techniques. The typical applications are introduced beneath and are also listed in Table 1.

3.1.1. Use of carbon nanomaterials in sample preparation. Carbon NMs are the most popular NMs in environmental analysis. In recent years, different strategies have been developed to modify and functionalize carbon NMs to overcome the hydrophobicity of the surface or to improve their stability and solubility in aqueous solutions. ${ }^{747}$ These strategies include oxidation, ${ }^{28,76,77}$ amination, ${ }^{78-81}$ carbonylation, ${ }^{78,82}$ hybridization, or modification with specific hydrophilic moieties, such as ionic liquids, ${ }^{83}$ silica, ${ }^{76} \mathrm{TiO}_{2},{ }^{84}$ polymers, ${ }^{50,77}$ and bismuth vanadate $\left(\mathrm{BiVO}_{4}\right){ }^{85}$ The resulting composite NMs offer effective tools for the analysis of drugs, phthalates, and endocrine disruptors in environmental samples.

Another recent trend in this area is the increasing use of molecular dynamics simulation tools to explore the adsorption mechanisms and to predict the affinity of different molecules for NMs. By using these tools, the performance and adsorption coefficients of chemical contaminants on activated carbon, charcoal, CNTs, and graphene could be predicted. ${ }^{75,86-89}$

Speltini et al. used amino-terminated alkyl-functionalized multi-walled carbon nanotubes (MWCNT- $-\mathrm{NH}_{2}$ ) as an SPE sorbent for the detection of perfluorooctanoic acid (PFOA) and perfluorooctane sulfonate (PFOS) followed by ultra-performance liquid chromatography-electrospray tandem mass spectrometry (UPLC-ESI-MS) in surface waters. ${ }^{90}$ Patiño et al. used MWCNTs, carboxyl-functionalized MWCNTs (MWCNT-COOH), aminofunctionalized MWCNTs (MWCNT- $\mathrm{NH}_{2}$ ), and nitrogen-doped CNTs (N-CNTs) as sorbents to extract three different types of compounds (i.e., a chlorinated paraffin 1,8-dichlorooctane, a quinolone nalidixic acid, and an alkylphenoletoxilate 2-(4methylphenoxy)ethanol). ${ }^{78}$ Comer et al. applied naked and hydroxylated CNTs for the adsorption of 30 small aromatic compounds with a variety of functional groups and also predicted the adsorption equilibrium constants by theoretical computation (Fig. 1). Among the different modified forms of graphenic carbon, the polyvinylpyrrolidone conjugation showed the highest affinity and could be selected as an efficient adsorbent for the sequestration of atrazine. ${ }^{91}$

For graphene, commercially available GO, ${ }^{92}$ colloidal GO nanoparticles, ${ }^{93}$ GO-based hydrogels, ${ }^{94}$ ionic liquids-GO composites, ${ }^{83}$ and thermo-responsive GO-perylene bisimidescontaining poly( $N$-isopropylacrylamide) hybrid $^{95}$ have been developed for the adsorption of drugs, phthalates, and organic pollutants from water samples. GO is commonly used as a starting material to synthesize other graphene-based composite adsorbents. Al-Khateeb et al. reported a method whereby graphene nanoplatelets were directly used as an adsorbent to remove aspirin, caffeine, and acetaminophen from Red Sea water, waste water, and tap water. ${ }^{96}$ Sun et al. prepared a miniaturized self-assembled graphene pipette-tip solid-phase extraction (PT-G-SPE) cartridge, and then the cartridge was coupled with liquid chromatography-fluorescence detection (LC-FD) to enrich and detect three sulfonamide antibiotics in 


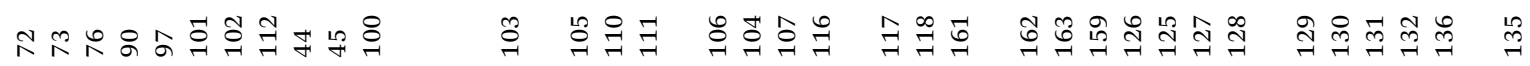

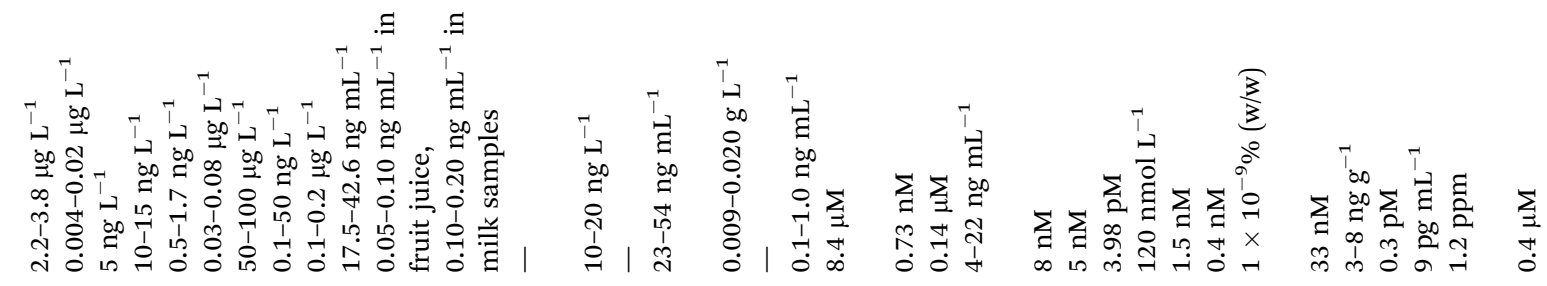

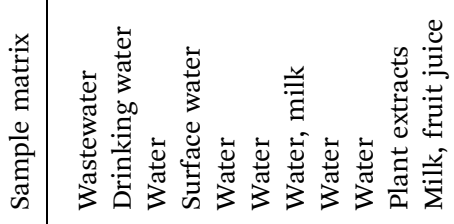

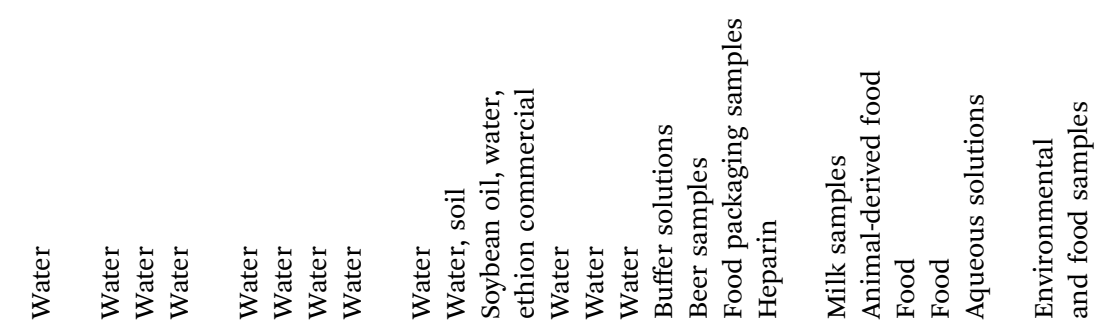

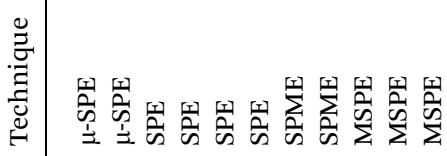

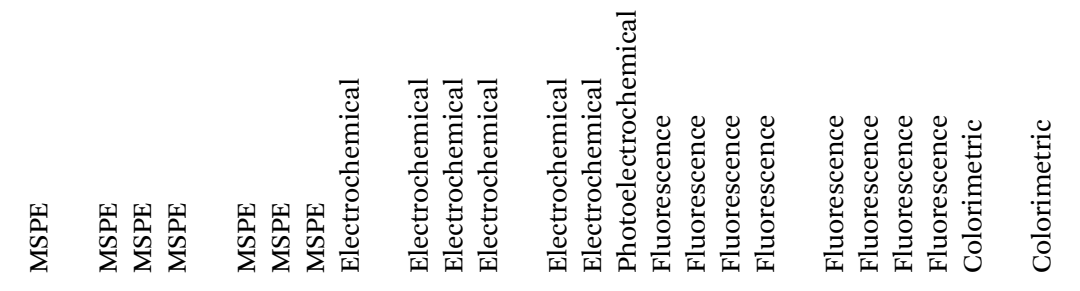
(1)
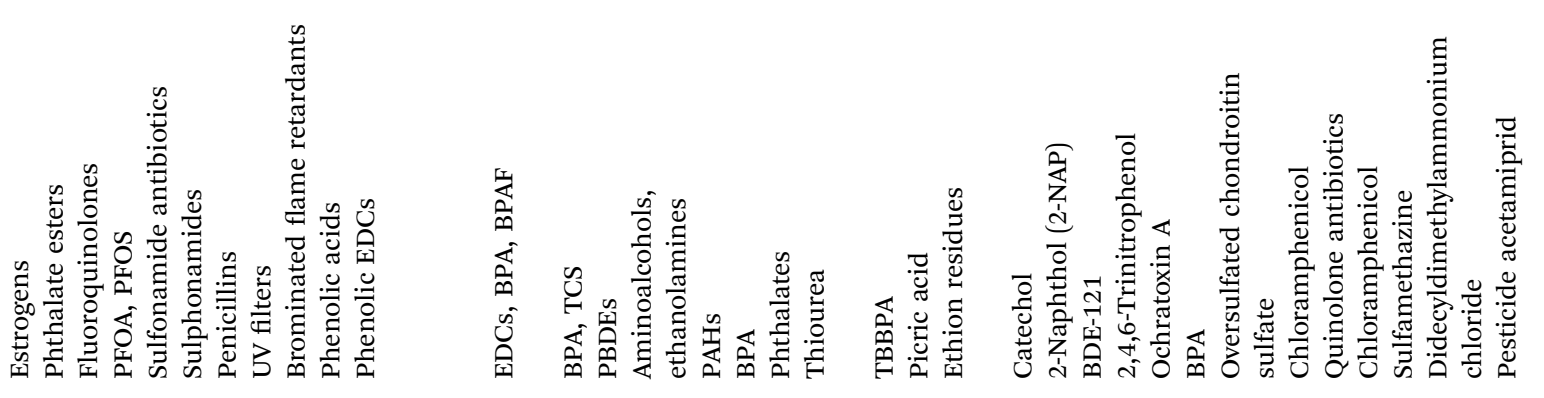

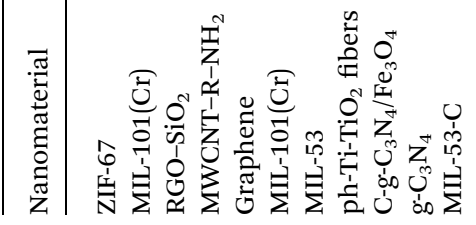

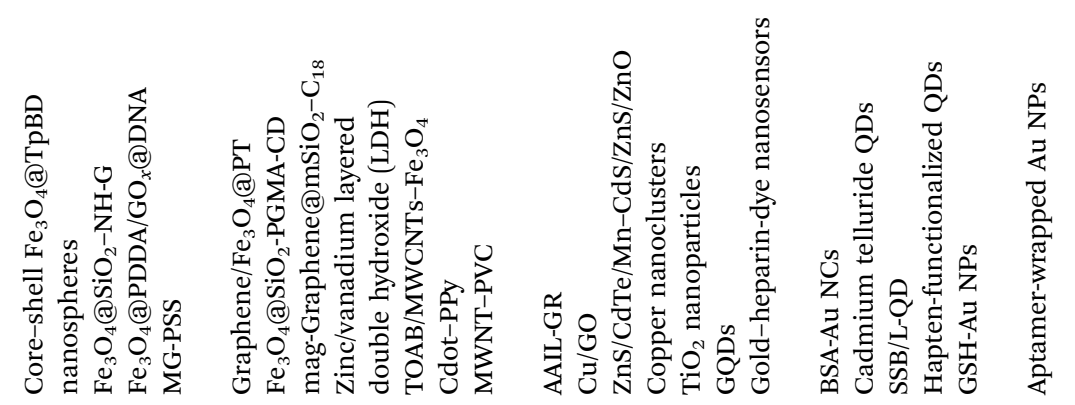




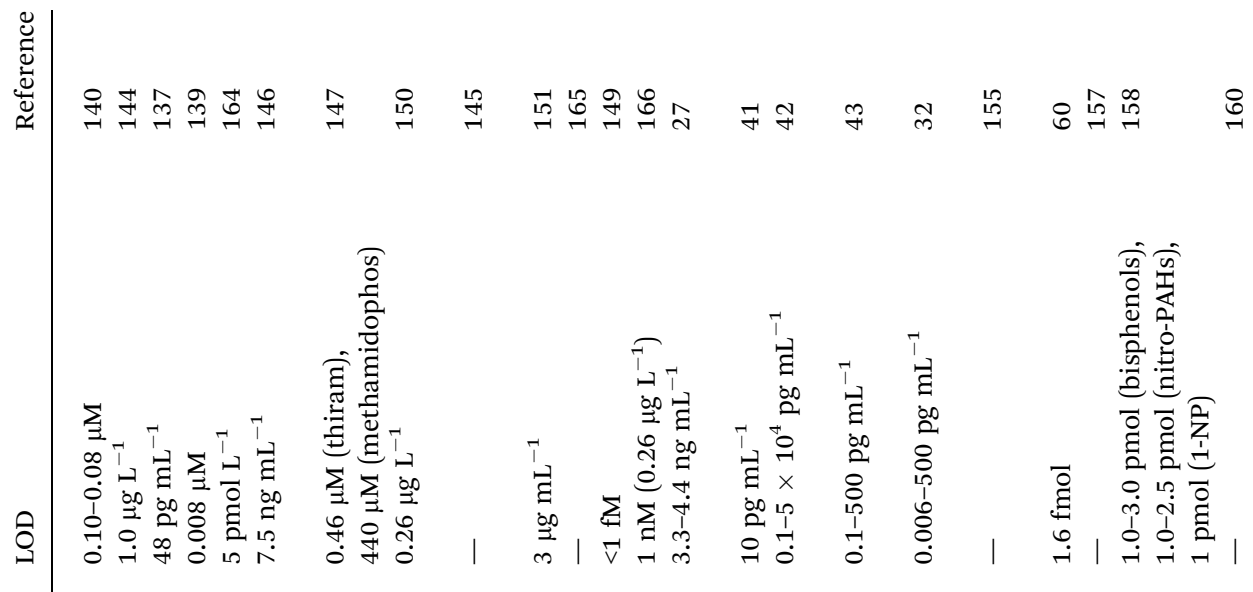

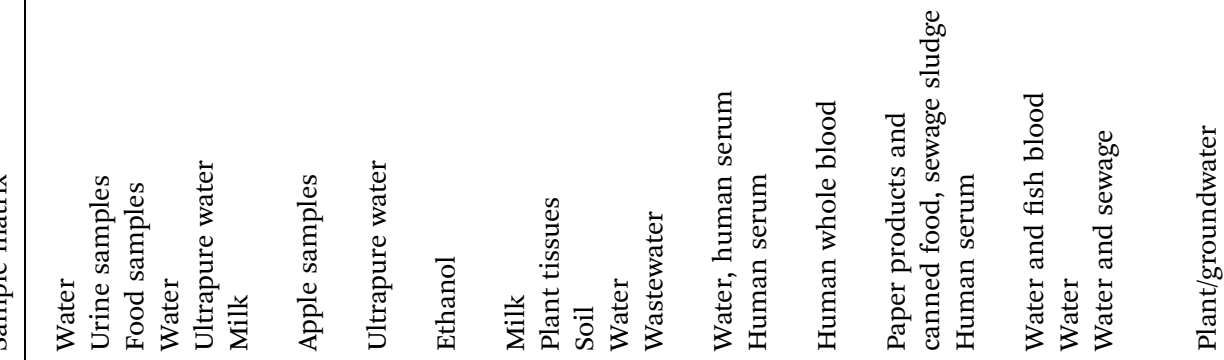

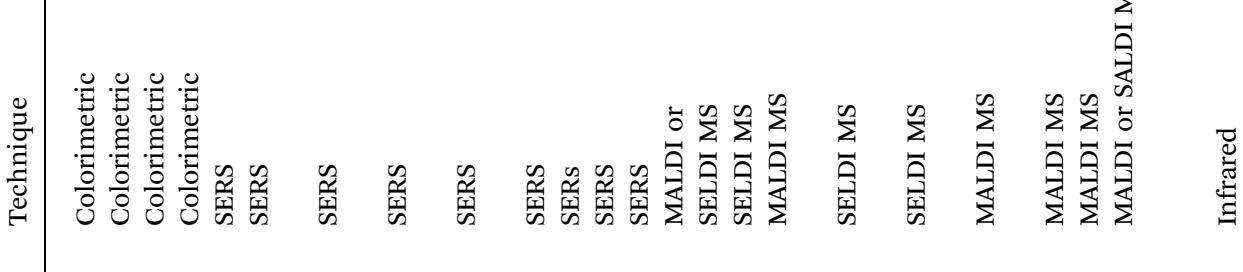

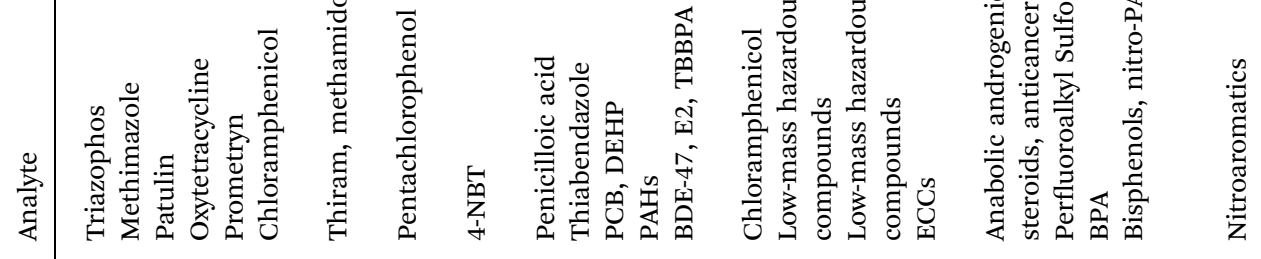

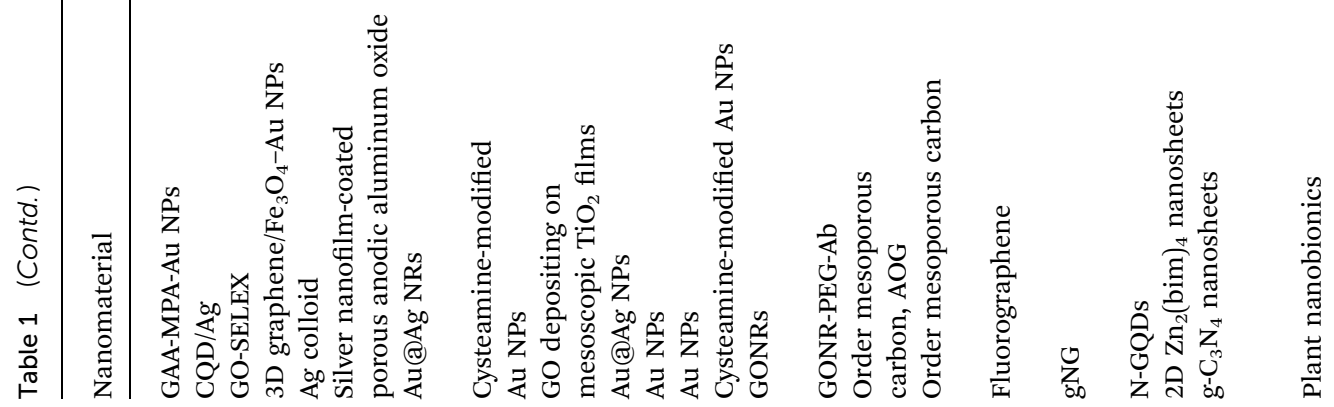



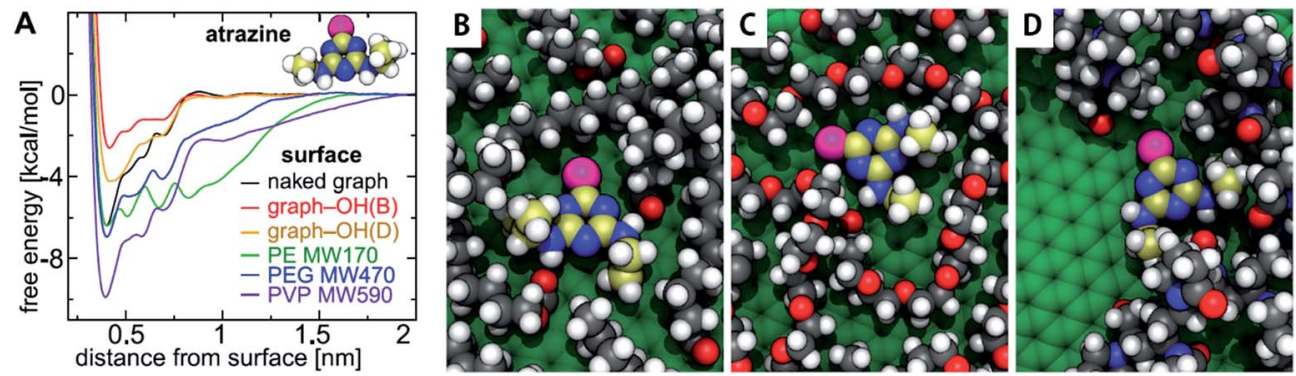

Fig. 1 Adsorption of the herbacide atrazine on functionalized graphenic surfaces. (A) Free energy as a function of distance between an atrazine molecule and the hydroxylated and polymer-conjugated graphene surfaces. (B-D) Representative simulation snapshots of atrazine adsorbed on graphene surfaces conjugated with PE, PEG, and PVP, respectively. For contrast, $C$ atoms of the polymers and graphene are shown in gray and green, respectively. ${ }^{91}$ Copyright (2015) American Chemistry Society.

environmental water samples. ${ }^{97}$ Chen et al. developed an approach to remove sulfamethoxazole and ciprofloxacin from water samples using GO as an adsorbent. ${ }^{98}$

For graphene-based composites, Speltini et al. prepared a hybrid material consisting of silica microparticles modified with graphene (RGO-silica). The RGO-silica was used as a mixed-mode sorbent for the preconcentration of fluoroquinolones in water samples based on SPE followed by HPLCFD detection. The method was applied in real environmental

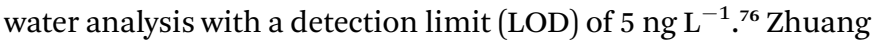
et al. applied a regenerable $\mathrm{TiO}_{2}$ nanotube/reduced graphene oxide (RGO-TON) hydrogel to improve the performance of the adsorption and removal of ciprofloxacin from aqueous solutions. ${ }^{84}$ Chen et al. explored the multiple adsorption mechanisms of nitro-aromatic compounds on GO, reduced graphene oxide (RGO), and graphene nanosheets by an infrared spectroscopy technique, demonstrating that $\pi-\pi$ electron donoracceptor interactions, charge electrostatic, and polar interactions were responsible for the adsorption. ${ }^{89}$

3.1.2. Use of MOFs in sample preparation. Inspired by their porous structure and excellent adsorption capacity, MOFs have been developed as sorbents for the sample preparation of ECCs in different techniques, including SPE, SPME, MSPE, and $\mu$-SPE. ${ }^{72,73}$ As mentioned above, since the prerequisite for the application of MOFs in environmental analysis is stability in water, several water-stable MOFs have been prepared to meet this requirement, such as ZIF-8, ZIF-67, MIL-100(Fe), and UiO$66(\mathrm{Zr})$, in the extraction of phthalate esters, drugs, endocrinedisrupting compounds, and other contaminants. ${ }^{53-56,99}$

Liu et al. used Fe-MIL-53 as a sorbent for the preconcentration of four phenolic EDCs in fruit juices and milk samples based on the strong $\pi-\pi$ stacking interaction. The target contaminants were detected by high-performance liquid chromatographic-ultraviolet detection (HPLC-UV) with LODs of 0.05-0.10 ng mL $\mathrm{mL}^{-1}$ for fruit juice and $0.10-0.20 \mathrm{ng} \mathrm{mL}^{-1}$ for milk samples. ${ }^{\mathbf{1 0 0}}$

Dai et al. developed a novel method to extract four sulphonamides from environmental water followed by UPLC-MS/MS analysis. MIL-101 was used as an SPE adsorbent due to the large specific surface area, high porosity, and excellent chemical stability. Under the optimized conditions, the LODs of the target analytes could reach $0.03-0.08 \mu \mathrm{g} \mathrm{L}{ }^{-1} .^{101}$
MOFs could also be integrated into an SPME monolithic column. Lirio et al. fabricated an aluminum-based MIL-53organic polymer monolithic column and applied it as an SPME sorbent for the extraction of trace amounts of penicillin in river water and milk. The LOD was $50 \mu \mathrm{g} \mathrm{\textrm {L } ^ { - 1 }}$ in river water and $100 \mu \mathrm{g} \mathrm{\textrm {L } ^ { - 1 }}$ in the milk samples. ${ }^{\mathbf{1 0 2}}$

González et al. fabricated $\mu$-SPE devices by using MOFs as sorbents. They used MIL-101(Cr) to pre-concentrate a trace amount of six phthalate esters in drinking water coupled with GC-MS analysis. The theoretical computational data from molecular modeling was combined with the experimental results to predict the extraction efficiencies, demonstrating this as a promising tool for the analysis of trace amounts of ECCs. $^{72}$

Li et al. recently reported a monomer-mediated in situ growth strategy for the controllable synthesis of core-shell nanocomposites with a magnetic $\mathrm{Fe}_{3} \mathrm{O}_{4}$ core and a COF shell. ${ }^{103} \mathrm{~A}$ highly stable porous $\mathrm{COF}, \mathrm{TpBD}$ (Tp and $\mathrm{BD}$ refer to 1,3,5-triformylphloroglucinol and benzidine, respectively), as a shell showed a fast adsorption of typical environmental endocrinedisrupting chemicals, namely bisphenol A (BPA) and bisphenol AF (BPAF), in aqueous solutions.

3.1.3. Use of magnetic nanomaterials in magnetic solidphase extraction. MSPE has been wildly applied in the enrichment and removal of ECCs. The development of magnetic nanoadsorbents is a highly active research area. Recently, magnetic $\mathrm{Fe}_{3} \mathrm{O}_{4}$ functionalized with $\beta$-cyclodextrin ( $\beta$-CD) and $\mathrm{C}_{3} \mathrm{~N}_{4}$ was applied in the analysis of ECCs based on host-guest recognition and hydrophobic interactions. ${ }^{\mathbf{1 0 4}}$ The magnetic particles were typically encapsulated with silica or polymer shells to prevent them from being oxidized and also to facilitate further modification. ${ }^{45,104-107}$ Ionic liquids, ${ }^{108}$ MIPs, ${ }^{50}$ and MOFs $^{109}$ have been used to functionalize magnetic materials.

Zhou et al. synthesized MWCNTs coated with magnetic amino-modified $\mathrm{CoFe}_{2} \mathrm{O}_{4}\left(\mathrm{CoFe}_{2} \mathrm{O}_{4}-\mathrm{NH}_{2}\right)$ nanoparticles and used them as adsorbents for the adsorption of tetrabromobisphenol A (TBBPA) in water samples. ${ }^{51}$

Wang et al. fabricated ferromagnetic $\mathrm{Fe}_{3} \mathrm{O}_{4} @ \mathrm{SiO}_{2}-$ poly (glycidyl methacrylate)- $\beta$-CD-nanoparticles by a radical polymerization, core-shell package, and graft-through strategy. Combining the ferromagnetism of $\mathrm{Fe}_{3} \mathrm{O}_{4}$ and the host-guest recognition between $\beta$-CD and aromatic molecules, the hybrid 
nanoparticles could be used to quickly eliminate, extract, and detect bisphenol A (BPA). ${ }^{104}$

Yang et al. deposited magnetite $\left(\mathrm{Fe}_{3} \mathrm{O}_{4}\right)$ particles onto a graphitic carbon nitride $\left(\mathrm{g}-\mathrm{C}_{3} \mathrm{~N}_{4}\right)$ nanosheet surface by a facile in situ growth method. The as-synthesized NMs were applied in the separation of phenolic acids by MSPE based on the high affinity of $\mathrm{g}-\mathrm{C}_{3} \mathrm{~N}_{4}$ for phenolic acids. The simultaneous detection of protocatechuic, caffeic, and ferulic acids in Salicornia herbacea L. plant extracts was achieved using this material. ${ }^{45}$ Wang et al. reported a new strategy for the MSPE of brominated flame retardants (BFRs) from a water sample using a 2D magnetic porous carbon-doped graphitic carbon nitride nanocomposite $\left(\mathrm{C}-\mathrm{g}-\mathrm{C}_{3} \mathrm{~N}_{4} / \mathrm{Fe}_{3} \mathrm{O}_{4}\right)$. ${ }^{44}$

Zang et al. synthesized graphene-grafted magnetic ferroferric oxide microspheres $\left(\mathrm{Fe}_{3} \mathrm{O}_{4} @ \mathrm{SiO}_{2}-\mathrm{NH}-\mathrm{G}\right)$. This material was applied in the adsorption of BPA and triclosan (TCS) from water samples by MSPE followed by GC/MS. Under the optimized conditions, the LODs of BPA and TCS were 10.0 and $20.0 \mathrm{ng} \mathrm{L}^{-1}$, respectively. ${ }^{105}$

Gan et al. prepared novel magnetic $\mathrm{Fe}_{3} \mathrm{O}_{4} @ P D D A / \mathrm{GO}_{x} @ D N A$ nanoparticles (PDDA = poly(diallyldimethyl ammonium chloride)) by hydrothermal procedures and a layer-by-layer selfassembly method. The nanoparticles were used as adsorbents to extract and remove polybrominated biphenyls (PBDEs) from real water samples based on MSPE followed by GC/MS. The LODs were at the ppb levels. ${ }^{110}$

Huang et al. fabricated magnetic graphene/mesoporous silica composites with $\mathrm{C}_{18}$-modified pore-walls (mag-graphene@ $\mathrm{mSiO}_{2}{ }^{-}$ $\mathrm{C}_{18}$ ) by a surfactant-mediated co-condensation sol-gel process. The as-prepared nanocomposites were applied in the enrichment of six typical phthalates and investigation of their extraction abilities. Using GC/MS analysis, the resulting LODs were in the range of $0.1-10 \mathrm{ng} \mathrm{mL}{ }^{-1} \cdot{ }^{107}$ Chinthakindi et al. ${ }^{111}$ and Mehdinia et al. ${ }^{106}$ used polystyrene sulfonic acid and polythiophene (PT) to modify magnetic graphene using non-covalent wrapping methods. The former nanocomposites were dispersed in water as a cation exchange sorbent for the extraction of amino alcohols and ethanolamines. ${ }^{111}$ The latter was used as an MSPE adsorbent for the enrichment of polycyclic aromatic hydrocarbons (PAHs) in environmental water samples. ${ }^{106}$

3.1.4. Use of $\mathrm{TiO}_{2}$ nanoparticles in sample preparation. $\mathrm{TiO}_{2}$ nanoparticles are mainly used as SPME sorbents for the extraction of ECCs owing to their excellent chemical stability, durability, corrosion resistance, non-toxicity, and cost effectiveness. ${ }^{70}$ In addition, $\mathrm{TiO}_{2}$ nanoparticles can be used to remove ECCs from waters by photocatalytic degradation. ${ }^{69-71}$

Li et al. prepared a $\mathrm{TiO}_{2}$ nanocoating by one-step anodization on a titanium wire substrate in ethylene glycol and then further functionalized it with phenyl groups. The hybrid nanofiber was applied as an SPME sorbent for the preconcentration of several UV filters, such as (2-hydroxy-4-methoxybenzophenone (BP-3), 2ethylhexyl-4-methoxycinnamate (EHMC), 2-ethylhexyl 4-( $N, N$ dimethylamino), benzoate (OD-PABA), and 2-ethylhexyl salicylate (EHS)), coupled with HPLC-UV. The method could determine a low concentration of UV filters with LODs of $0.1-50 \mathrm{ng} \mathrm{L}^{-1}$. $^{112}$

In addition to the NMs above-mentioned, Brandl et al. prepared a photocleavable amphiphilic diblock poly(ethylene glycol)-b-poly(lactic acid) (PEG- $b$-PLA) copolymer and used it to fabricate a novel platform of photosensitive core-shell nanoparticles for the extraction of hydrophobic pollutants from water and contaminated soil. Furthermore, the method was successfully applied in the treatment of BPA, TCS, and $17 \alpha-$ ethinyl estradiol to reduce toxicity in wastewater, thermal printing paper, and contaminated soil in in vivo experiments. ${ }^{113}$

Singla et al. fabricated boron nitride (BN) NMs with two different morphologies by utilizing a solid-state hightemperature annealing approach. The as-prepared NMs were applied in the adsorption of ciprofloxacin (CIP) and norfloxacin (NOR). In addition, the materials were shown to be a potentially useful for water purification. ${ }^{114}$

Schiwy et al. used a zero-valent iron nanomaterial to eliminate halogenated pollutants at contaminated sites and assessed the toxicity in anaerobic batch and column experiments. ${ }^{115}$

\subsection{Electrochemical sensing}

For the analysis of ECCs, electrochemical methods provide the possibility of in-site, real-time approaches to monitor the ECCs in the environment. Nanoscale electrode materials control the sensitivity and selectivity of the sensors based on the size effect and high electron transfer area. ${ }^{116}$ Different electrode materials and electrode surfaces have been designed to adsorb ECCs and to enhance the electron transfer and electrochemical signals.

Zhou et al. developed an electrochemical sensor to sensitively determine TBBPA in water samples. The sensor was fabricated using a trimethyloctadecylammonium bromide and MWCNT- $\mathrm{Fe}_{3} \mathrm{O}_{4}$ hybrid (TOAB/MWCNTs- $\mathrm{Fe}_{3} \mathrm{O}_{4}$ ). It was demonstrated that the electrochemical response of TBBPA could be greatly enhanced by the combined use of MWCNTs$\mathrm{Fe}_{3} \mathrm{O}_{4}$ and TOAB, and the LOD of TBBPA reached $0.73 \mathrm{nM}$, with good reproducibility and stability. ${ }^{117}$

Combining the excellent conductivity properties of carbon dots with the flexible structures of polymers, Pal et al. prepared carbon dot-polypyrrole (Cdot-PPy) nanocomposites with higher electrical conductivity than pure carbon dots or PPy, and then fabricated a device for the electrochemical sensing of chemical contaminants. The synthetic process of the nanocomposites and the results from the device obtained in a current-voltage (IV) characteristics study is depicted in Fig. 2. Based on this device, they developed a method for on-the-spot sensitive and selective detection of picric acid and ethylene diamine in water and soil. The LOD was $1.40 \times 10^{-7} \mathrm{M}(32 \mathrm{ppb}) .{ }^{118}$

Khan et al. prepared a vanadium and zinc layered double hydroxide (LDH) and used it to modify the surface of a silver electrode. The sensor was applied in the detection of thiourea in water samples with high sensitivity $\left(1.004 \mu \mathrm{A} \mu \mathrm{M} \mathrm{cm} \mathrm{cm}^{-2}\right)$, low LODs (8.4 mM), and high selectivity. ${ }^{116}$

\subsection{Fluorescence detection}

NMs can be used as either fluorophores or quenchers in designing fluorescent detection systems. QDs, such as traditional metallic QDs (CdTe, ZnS), carbon dots, ${ }^{68}$ graphene QDs, and silicon QDs, ${ }^{119,120}$ have been used as fluorophores to determine ECCs based on their unique physicochemical, 


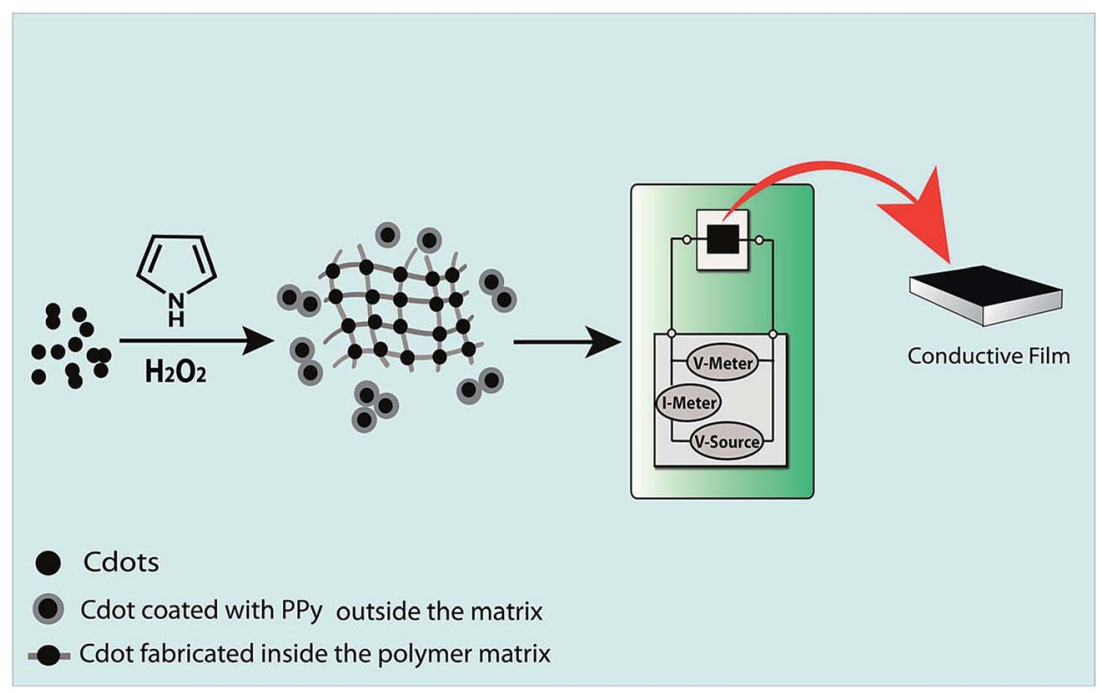

Fig. 2 Schematic representation of the synthesis of Cdot-PPy nanocomposites and the experimental set-up for the I-V characteristic study of the composite film. The Cdot-PPy composites were prepared by repulsive polymerization of Cdots and pyrrole in $\mathrm{HCl}$ medium. The nanocomposites were assembled into a conductive film to develop an electrochemical device for the detection of picric acid. ${ }^{118}$ Copyright (2016) American Chemistry Society.

electronic, magnetic, and optical properties. Recently, due to the low toxicity and biocompatibility, upconversion NMs have also become a popular choice for the online, in situ, nondestructive monitoring of ECCs based on fluorescence detection. For example, $\mathrm{NaYF}_{4}: \mathrm{Yb} / \mathrm{Tm}, \mathrm{KGdF}_{4}: \mathrm{Tb}^{3+}$, and $\mathrm{YVO}_{4}: \mathrm{Eu}^{3+}$ nanoparticles were prepared and used as fluorophores. ${ }^{\text {121-124 }}$ Furthermore, new NMs-based quenchers, such as $\mathrm{TiO}_{2}$ nanoparticles ${ }^{125}$ and copper nanocluster, ${ }^{126}$ have also been reported in addition to the previously used Au NPs and GO.

Huang et al. developed a novel method for the detection of BPA by quenching the fluorescence of graphene QDs (GQDs) in the presence of horseradish peroxidase and the oxidation of BPA. The method was successfully applied in the analysis of real food packaging samples with an LOD of $0.4 \mathrm{nM}^{.27}$

Kalita et al. synthesized gold-heparin-dye nanosensors and demonstrated strong dye-induced fluorescence quenching by nanometal surface energy transfer (NSET). Based on this mechanism, they developed a rapid and sensitive method for the detection of oversulfated chondroitin sulfate (OSCS) contaminant in heparin. The LOD was $1 \times 10^{-9} \%(\mathrm{w} / \mathrm{w})$ and the method was successfully used to determine trace amounts of OSCS in pharmaceutical heparins. ${ }^{\mathbf{1 2 8}}$

Tan et al. developed a sensor for chloramphenicol (CAP) based on the photo-induced electron transfer between CAP and bovine serum albumin-stabilized $\mathrm{Au}$ nanoclusters (BSA-Au NCs). The LOD of CAP was $33 \mathrm{nM}$ in milk samples. ${ }^{129}$

$\mathrm{Hu}$ et al. prepared $\mathrm{NaYF}_{4}: \mathrm{Yb} / \mathrm{Tm}$ upconversion nanoparticles and monodispersed magnetic polystyrene microspheres (MMPMs). The upconversion nanoparticles and MMPMs were modified with antibodies and coating antigen, respectively. Using the former as a fluorescence probe and the latter as an immune-sensing capture probe, they developed a novel competitive fluorescence immunoassay for the determination of sulfaquinoxaline (SQX) with an LOD of $0.1 \mu \mathrm{g} \mathrm{L}^{-1}$ in buffer and $0.5 \mu \mathrm{g} \mathrm{kg}{ }^{-1}$ in the animal-derived food samples. The method was successfully applied in the analysis of SQX in milk and animal-derived foods. ${ }^{\mathbf{1 2 2}}$

Sharma et al. developed an aptasensing platform based on $\mathrm{TiO}_{2}$ nanoparticles-induced quenching of the fluorescence of a fluorescein-labeled aptamer. The method was applied in the detection of ochratoxin A in beer samples. The mechanism of the $\mathrm{TiO}_{2}$ nanoparticles-induced fluorescence quenching and recovery was related to the non-covalent adsorption of aptamer onto the surface of $\mathrm{TiO}_{2}$ nanoparticles and the formation of the aptamer G-quadruplex by the introduction of ochratoxin A. This method showed several advantages, such as high sensitivity (the LOD was $1.5 \mathrm{nM}$ ), good selectivity, and less background interference. ${ }^{125}$

Based on fluorescence resonance energy transfer (FRET) between lanthanide-doped $\mathrm{KGdF}_{4}$ nanoparticles $\left(\mathrm{KGdF}_{4}: \mathrm{Tb}^{3+}\right.$ NPs) and Au NPs, Duan et al. developed a method for the detection of BPA. The lanthanide-doped $\mathrm{KGdF}_{4}$ and Au NPs were modified with an aptamer and the complementary DNA (cDNA) of the aptamer, respectively. ${ }^{\mathbf{1 2 4}}$

Meng et al. reported a new fluorescence assay for the detection of five quinolone antibiotic residues in animal-derived food samples by using micellar electrokinetic capillary chromatography (MEKC) and QDs. The LODs were 3-8 $\mathrm{ng} \mathrm{g}^{-1}$, which could meet the maximum residue limits required by the European Union and Japan. ${ }^{\mathbf{1 3 0}}$ Liu et al. prepared surface molecularly imprinted $\mathrm{YVO}_{4}: \mathrm{Eu}^{3+}$ nanoparticles $\left(\mathrm{YVO}_{4}: \mathrm{Eu}^{3+} @ \mathrm{MIPs}\right)$ by precipitation polymerization using $\lambda$-cyhalothrin as a template molecule. On the basis of the fluorescence signal of lanthanidedoped $\mathrm{YVO}_{4}: \mathrm{Eu}^{3+}$ and the selective recognition ability of MIPs, they developed a method for the detection of the residues of $\lambda$ cyhalothrin in real examples, with an LOD of $1.76 \mu \mathrm{M} .{ }^{123}$ Miao et al. described a strategy for the detection of CAP residues in food samples based on FRET between labeled single-stranded DNA binding protein (SSB)-limposome-CdSe/ZnS quantum dot 


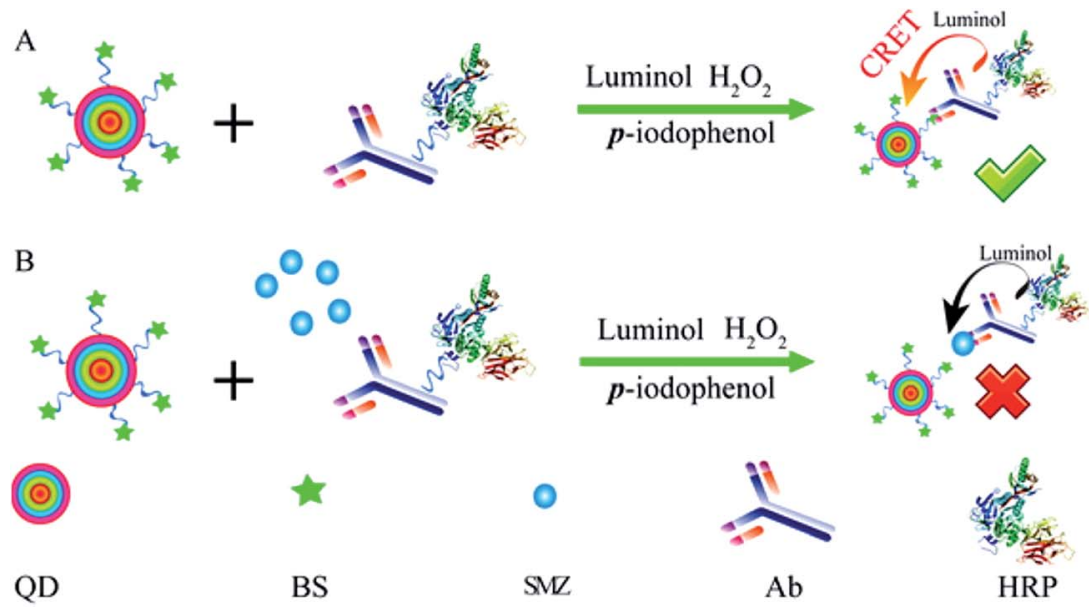

Fig. 3 (A) Schematic illustration of the CRET process between luminol and QDs based on the immunoreaction between mAb-HRP and BS-QDs. (B) Schematic illustration of the CRET-based competitive immunoassay for sulfamethazine (SMZ). ${ }^{132}$ Copyright (2016) American Chemistry Society.

(SSB/L-QD) complexes and aptamer-labeled nano-gold (Au-Apt). The probe could be used to detect CAP in the milk samples by fluorescence "off-on", with an LOD down to 0.3 pM. ${ }^{131}$

Besides FRET, chemiluminescence resonance energy transfer (CRET) is another useful technique for the analysis of ECCs. In comparison with FRET, CRET has some advantages, such as low autofluorescence background, nonbleaching emission, and no external light source. Ma et al. assembled a CRET-based competitive immunoassay by using the hapten molecule (BS) and mAb-HRP (horseradish peroxidase linked to specific antibody (mAb4D11)) to modify amphiphilic polymer-modified core/ multishell QDs. With this material, they developed a method for the determination of sulfamethazine (SMZ) as the addition of SMZ could inhibit the fluorescence intensity increasing via CRET, where the addition of luminol was used as a donor and BS-QDs as the acceptor. The detailed mechanism is shown in Fig. 3.132

\subsection{Colorimetric detection}

Colorimetric detection has the advantages of simplicity, low cost, rapidity, and the possibility for naked eye detection. In recent years, $\mathrm{Au}$ and $\mathrm{Ag}$ NPs have remained the most important nanoprobes in colorimetric detection. However, graphene and carbon dots have now emerged as novel probes in colorimetric detection. Prior to use, nanoprobes are commonly functionalized by organic or biological molecules, such as dithiocarbamate- $p$-tertbutylcalix[4] arene, ${ }^{133} \beta-\mathrm{CD},{ }^{134}$ aptamers, ${ }^{135}$ or glutathione (GSH), ${ }^{136}$ to enhance the stability of the probes and endow the probes with recognition ability for specific target analytes. The addition of target analytes can change the interparticle distance of the functionalized nanoprobes to cause a shift in the UV-visible spectra. Colorimetric detection has recently been applied in the analysis of acetamiprid, patulin, metsulfuronmethyl, didecyldimethylammonium chloride, oxytetracycline (OTC), antibacterial, and so on. ${ }^{\mathbf{1 3 4 - 1 4 4}}$

Kim et al. used GSH-functionalized Au NPs as a colorimetric sensor to determine the disinfectant didecyldimethylammonium chloride (DDAC). The target analyte could be detected by naked eye within $10 \mathrm{~min}$ or by UV-visible spectrometry, with an LOD as low as $1.2 \mathrm{ppm}$. Furthermore, the sensor was successfully applied in the real-time detection of DDAC in aqueous solutions. ${ }^{\mathbf{1 3 6}}$

Amjadi et al. prepared glucose-derived carbon QDs and a silver nanocomposite by a simple and fast method utilizing microwaves. The color of the mixture of the as-prepared nanocomposite and anti-thyroid drug methimazole could sharply change from yellow to red upon their interaction. Based on this phenomenon, they developed a colorimetric sensor for methimazole with an LOD of $1.0 \mu \mathrm{g} \mathrm{L}^{-1}$ and applied it in the analysis of urine samples. ${ }^{\mathbf{1 4 4}}$

Wu et al. screened a high-affinity ssDNA aptamer for patulin (PAT-11) by a systemic evolution of ligands by exponential enrichment (SELEX) process assisted by GO. During the SELEX process, GO was applied on solid substrates for an in vitro separation step to adsorb unbound sequences from release sequences bound to the target via $\pi-\pi$ stacking interactions between ssDNA and GO. Using the selected aptamer and squaric acideiron(III) chelate, they fabricated an enzymechromogenic substrate system and further developed a method for the colorimetric detection of patulin in food samples. The method was applied in the analysis of patulin with high sensitivity and selectivity due to the high affinity and specific recognition of the aptamer for the target small molecule. The LOD was $48 \mathrm{pg} \mathrm{mL} \mathrm{m}^{-1}$. Fig. 4 shows the procedures for the GO-SELEX of the aptamer against patulin. ${ }^{137}$

Yuan et al. fabricated a $3 \mathrm{D}$ graphene $/ \mathrm{Fe}_{3} \mathrm{O}_{4}-\mathrm{Au}$ NPs. Compared with the traditional 2D graphene-based monometallic composites, the as-prepared 3D structure showed switchable peroxidase-like activity and a higher affinity for the substrate. By joint use with the aptamer, they constructed a colorimetric probe to detect oxytetracycline (OTC) without any labeling or chemical modification. The LOD was $8 \mathrm{nM} \cdot{ }^{139}$

\subsection{Surface-enhanced Raman spectroscopy}

SERS is a promising "fingerprint" technique for environmental analysis owing to its simplicity, rapidity, excellent sensitivity, good selectivity, and non-destructive properties. The research 

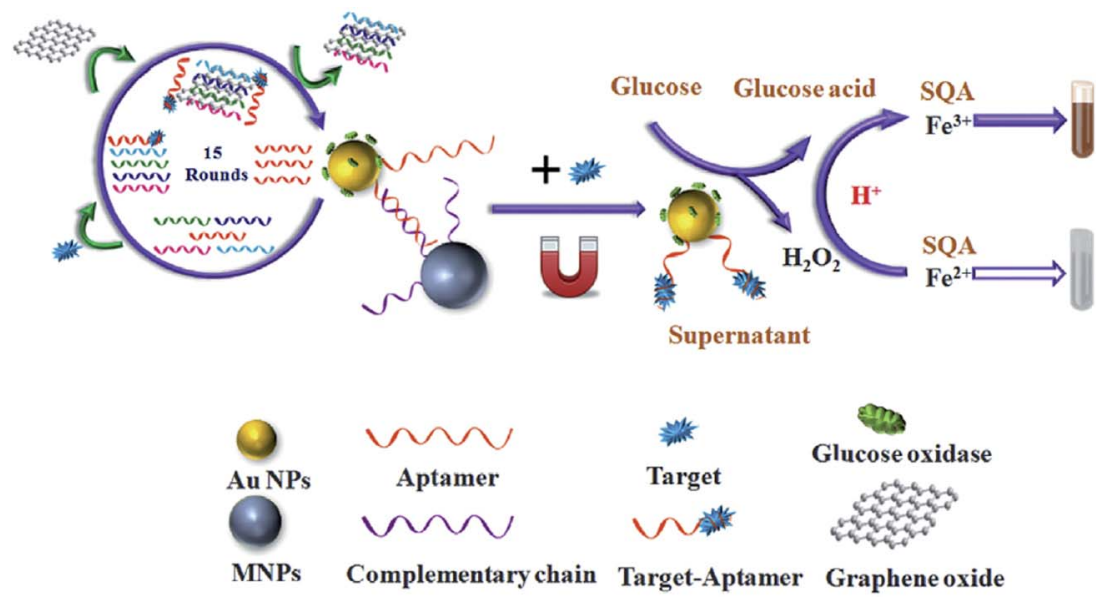

Fig. 4 Schematic illustration of aptamer selection against patulin using a GO-SELEX procedure and a colorimetric aptasensor for patulin detection. A high-affinity sSDNA aptamer for PAT-11 was selected by SELEX assisted by GO. Then, a colorimetric detection method was developed for patulin in apple juice based on the chromogenic reaction between glucose oxidase and squaric acid. ${ }^{137}$ Copyright (2016) Elsevier.

on SERS mainly focuses on the exploration of SERS substrates. In addition to the normally used $\mathrm{Au}$ and $\mathrm{Ag} \mathrm{NMs}, \mathrm{TiO}_{2}$ nanoparticles and GO were recently explored as SERS substrates. ${ }^{145}$

Chen et al. fabricated a 2D silver nanofilm-coated porous anodic aluminum oxide material (AAO) and used it as a SERSactive substrate in the detection of CAP in food samples. The material showed an excellent enhancement effect of the SERS signals with an enhancement factor of approximately $2 \times 10^{4}$. The sensor was applied in the quantification of CAP in milk, with an LOD of $7.5 \mathrm{ppb}^{146}$

Zhang et al. used gold@silver core-shell nanorods (Au@Ag NRs) to develop a rapid and straightforward method for the simultaneous SERS determination of two pesticides (thiram and methamidophos) in apple samples. The LODs were $0.46 \mu \mathrm{M}$ for thiram and $440 \mu \mathrm{M}$ for methamidophos. ${ }^{147}$

Yang et al. developed a method to real-time and in situ monitor pesticide penetration by SERS mapping based on the $\mathrm{Au}$ NPs substrate. They monitored the penetration of two different types of pesticides, namely ferbam and thiabendazole, in spinach leaves and found that these pesticides behaved differently from the different internal SERS mapping signals. Furthermore, the method could be used to investigate the fate of pesticides in plant tissues in a non-destructive manner. The detailed process and mechanism are shown in Fig. $5 .{ }^{148}$

To extend the SERS application to non-aqueous media, Yang et al. fabricated an ultrasensitive Au NPs-based SERS platform, termed slippery liquid-infused porous surface-enhanced Raman scattering (SLIPSERS), for the enrichment and delivery of target analytes in both aqueous and non-aqueous fluids. The platform built a smooth and stable interface to remove pinning of the liquid contact line by forming a film of lubricating fluid on a micro/nanoporous substrate. Using this platform, they could detect various chemicals (rhodamine 6G), biological molecules (e.g., DNA, protein), and environmental contaminants (e.g., bis(2-ethylhexyl) phthalate (DEHP), 4-aminothiophenol (4-ATP), and polychlorinated biphenyls (PCBs)) in different phases (liquid phase, gas phase, and solid phase) of samples by extraction and delivery in situ. The Au NPs-based SERS platform was used in the quantification of PCBs in water and soil samples. The LODs were at the subfemtomolar level $\left(<10^{-15} \mathrm{~mol} \mathrm{~L}^{-1}\right)$. The detailed process for the detection of PCBs in soil samples is shown in Fig. $6 .^{149}$

Ma et al. prepared cysteamine-modified Au NPs immobilized onto a glass surface by Au-S covalent bonds. The platform was used as a SERS substrate and subsequently permitted the highly sensitive detection of pentachlorophenol. The LOD was $1 \mathrm{nM}$ $\left(0.26 \mu \mathrm{g} \mathrm{L}^{-1}\right) .{ }^{150}$

Graphene-based materials have also been applied as SERS substrates. For example, Alam et al. constructed a SERS-active platform by using single- to few-layered GO deposited on mesoscopic $\mathrm{TiO}_{2}$ films to support Ag NPs. This 2D material provided an excellent sensing platform for the simultaneous detection of multiple target analytes due to the enrichment ability of RGO and the catalytic Ag NPs. The method was successfully applied to determine 4-nitrobenzenethiol (4-NBT) in environmental samples as well as catalyzing the transformation of 4-NBT to less toxic 4-aminobenzenethiol (4-ABT) in photocatalysis. ${ }^{145}$

Qi et al. described a strategy for the rapid determination of penicilloic acid (PA) in milk samples by a label-free SERS method. They found that the signals of SERS were affected by the core size and shell thickness of silver-coated gold nanoparticles (Au@Ag NPs)..$^{151}$

\subsection{Laser desorption/ionization mass spectrometry}

MALDI-TOF MS provides a simple and high-throughput tool for the analysis of biopolymers and large organic molecules. However, when it is applied to the analysis of small molecules, strong matrix interference in the low-mass region is usually observed. To overcome this problem, NMs, especially carbon NMs, have been explored as novel MALDI matrices due to their strong optical absorption properties, high energy-transfer efficiency, and high chemical stability to produce few ion fragments in the low-mass region. ${ }^{99}$ This makes the 

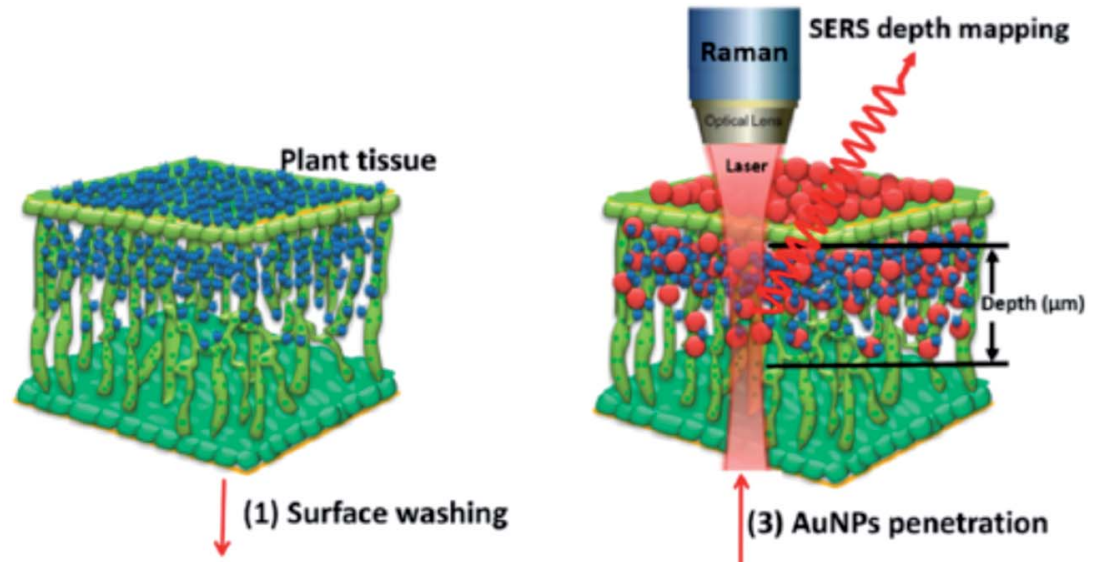

(3) AuNPs penetration

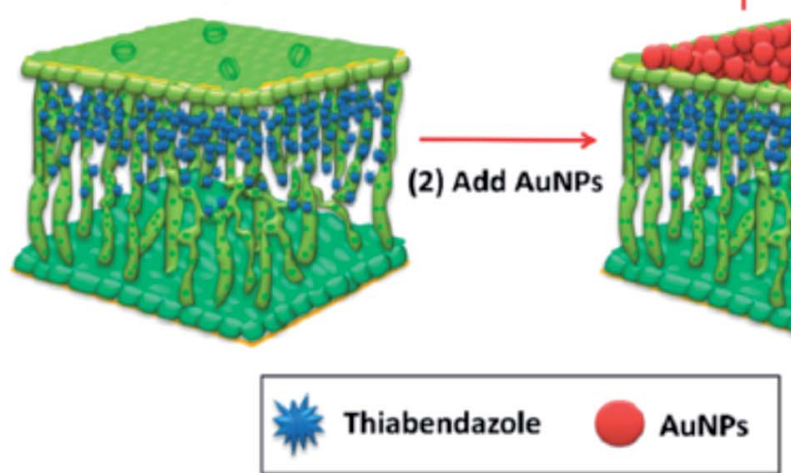

Fig. 5 Schematic illustration of the real-time and in situ monitoring of thiabendazole penetration in edible leaves by the SERS mapping technique using penetrative gold nanoparticles (Au NPs) as probes to enhance the internalized pesticide signals. ${ }^{148}$ Copyright (2016) American Chemistry Society.

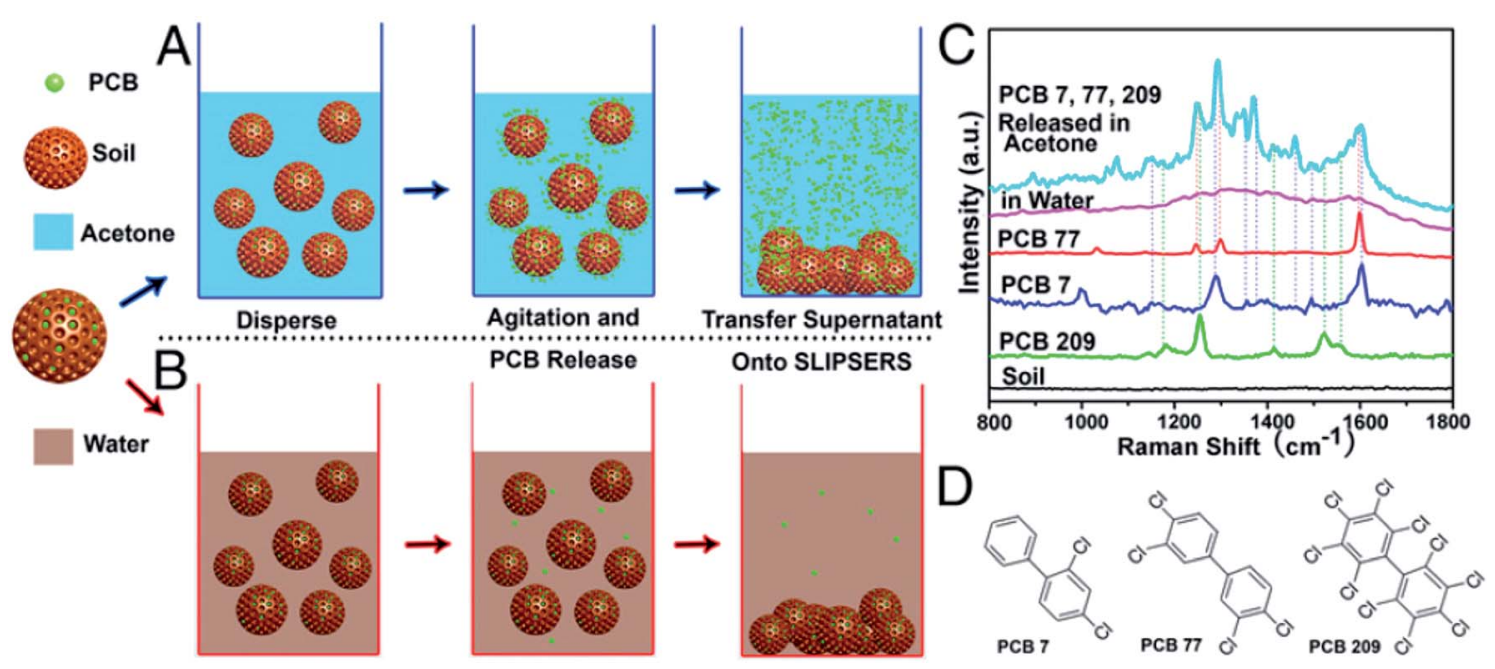

Fig. 6 Solid-phase detection of PCBs in contaminated soil using slippery liquid-infused porous surface-enhanced Raman scattering (SLIPSERS). (A) Using acetone as a dispersion liquid, PCB molecules can be released from the soil particulates. (B) Using water as a dispersion liquid, no detectable PCB molecules are released to the liquid medium. (C) Multiplex SERS detection of PCBs 7, 77, and 209 released from contaminated soil. (D) Molecular structures of PCBs 7, 77, and 209. ${ }^{149}$ Copyright (2016) PNAS.

high-throughput analysis of small molecules by MS possible. Furthermore, surface-enhanced laser desorption/ionization (SELDI), a variant mode of MALDI-TOF MS, has recently attracted much research interest. ${ }^{152-154}$ This technique uses a probe for the extraction, enrichment, desorption, and ionization of target analytes, thus greatly simplifying the analytical procedures and enhancing the sensitivity. NMs have been demonstrated to be useful probes for SELDI-TOF MS.

Liu et al. prepared graphene oxide nanoribbons (GONRs) by a one-step and one-pot pressurized oxidation of MWCNTs. Such 
a novel variety of graphene with a high length-to-width ratio and straight edges, as a matrix or probe in MALDI or SELDI MS, could produce significantly higher MS signals than other carbon NMs and allow the simultaneous detection of multiple small molecules (e.g., 2,2',4,4'-tetrabromodiphenyl ether (BDE47), estradiol, and TBBPA) in complex samples. The method was applied in the screening of ECCs in wastewater treatment plant samples. ${ }^{27}$

To further improve the specificity of the GONRs-based SELDI probe, Wang et al. functionalized GONRs with an antibody using polyethylene glycol (PEG) as a linker (see Fig. 7). The antibody could specifically bind the target analytes and the PEG could maintain the steric structure and activity of the antibody after it was bound to GONRs. This antibody-GONRs conjugate provided a highly selective and sensitive SELDI probe to detect chloramphenicol in river water and human serum samples. The

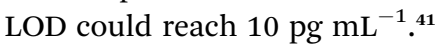

Wang et al. developed a two-step strategy for the MALDI-TOF MS screening of low-mass hazardous compounds in complex samples. In the first step, ordered mesoporous carbon was used to selectively extract the low-mass compounds while excluding the interference from large molecules in the sample matrix. In the second step, the analytes were eluted from the ordered mesoporous carbon and analyzed by MALDI-TOF MS with graphene as a matrix. The method was successfully applied in the rapid screening and identification of unknown toxic substances, such as perfluorochemicals, in human serum samples. ${ }^{\mathbf{4 2}}$

Huang et al. further investigated the use of ordered mesoporous carbon as a new MALDI matrix and SELDI probe in the detection of trace amounts of toxic chemicals in complex samples. With CMK-8 as a SELDI probe, they successfully identified and screened six perfluorinated compounds in a single drop of human whole blood without any complicated sample preparation procedure. The method provides a promising tool for high-throughput analysis of MS. ${ }^{43}$

Recently, Huang et al. prepared fluorographene (FG) by a one-step exfoliation of fluorographite. Based on the unique structure and self-assembly properties, FG was used as a MALDI matrix and SELDI probe with higher sensitivity and better reproducibility than other graphene-based materials. They showed the use of FG as a SELDI probe in the quantification of bisphenol $\mathrm{S}$ in paper products and high-fat canned food samples as well as in the identification and screening of quaternary ammonium halides in sewage sludge samples. ${ }^{32}$

Min et al. prepared a gas-phase N-doped graphene (gNG) by a modified thermal annealing method. The as-prepared material was used as a matrix for the analysis of anabolic androgenic steroids and anticancer drugs in negative ion MALDI-TOF MS. With the advantages of excellent salt tolerance and high sensitivity, the method provided a useful tool for the analysis of complex biological samples. ${ }^{155}$ Rao et al. also used N-doped graphene QDs (N-GQDs) as a matrix to detect perfluoroalkyl sulfonates and other small molecules in environmental samples by MALDI-TOF MS. ${ }^{156}$

Apart from graphene, other 2D nanosheets have also been used as SELDI probes in the analysis of small molecules. Liu et al. synthesized a water-stable $2 \mathrm{D}$ MOF nanosheet $\left(\mathrm{Zn}_{2}(\mathrm{bim})_{4}\right.$, bim = benzimidazole) by ultrasonic liquid exfoliation. By combining the advantages of MOFs and 2D nanosheets (e.g., good solubility, superior electronic and photonic properties), the material could be applied as a clean-background MALDI matrix for the analysis of small biomolecules and pollutants (e.g., BPA) in complex samples and in monitoring the hydrolysis of acetylcholine at ultra-high salt concentration. ${ }^{157}$

Lin et al. reported an ultrathin $\mathrm{g}-\mathrm{C}_{3} \mathrm{~N}_{4}$ nanosheet for the MALDI and surface-assisted laser desorption/ionization mass spectrometry (SALDI)-MS analysis of small molecules (e.g., amino acids, nucleobases, peptides, bisphenols, and nitroPAHs) without matrix interference. The matrix was used in the quantitative detection of 1-nitropyrene (1-NP) in sewage, with an LOD of 1 pmol. ${ }^{158}$

\subsection{Other techniques}

Li et al. prepared a core-shell ZnS/CdTe/Mn-CdS/ZnS sensitized macroporous ZnO nanosheet (NS) photoelectrode with an anti-
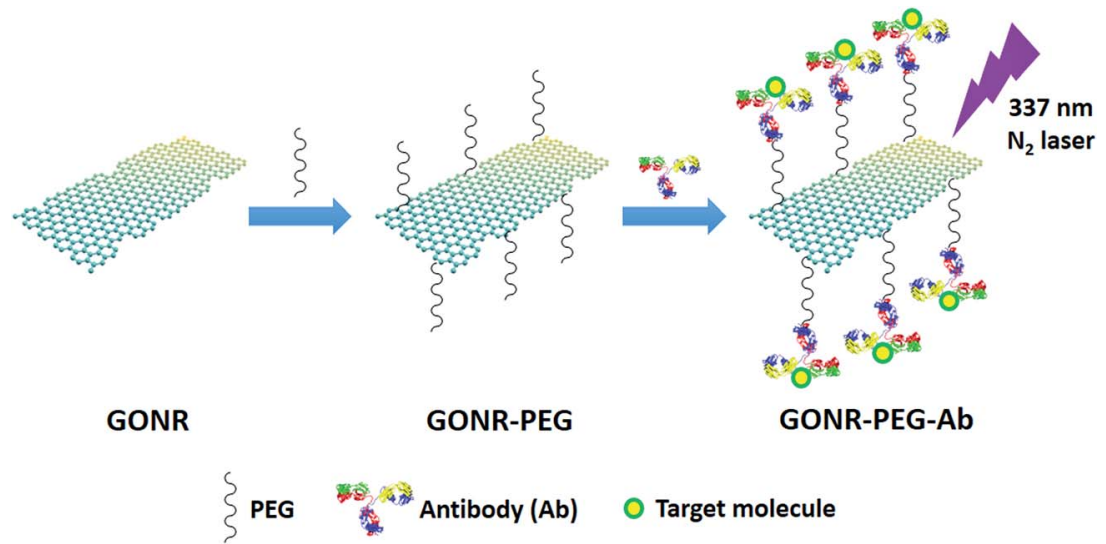

Fig. 7 Scheme showing the synthetic procedures of the graphene oxide nanoribbon-PEG-antibody (GONR-PEG-Ab) conjugate and its use as a probe in SELDI-TOF MS. N-(3-dimethylaminopropyl)- $N^{\prime}$-ethylcarbodiimide hydrochloride/N-hydroxysuccinimide (EDC/NHS) was utilized as a coupling agent to crosslink the amino end of $\mathrm{NH}_{2}-\mathrm{PEG}-\mathrm{COOH}$ and the carboxyl groups of GONRs. Then, the amino groups of the antibody were bound to the carboxyl end of the PEGylated GONRs. ${ }^{41}$ Copyright (2015) Royal Society of Chemistry. 
2,3',4,5',6-pentabromodiphenylether (BDE-121) polyclonal antibody coating. The as-prepared photoelectrode was used as a novel photoelectrochemical immunosensor to detect BDE121. The results indicated that the sensor was suitable for the rapid and sensitive detection of BDE-121, and its LODs was 3.98 pM. $^{159}$

Wong et al. reported a novel method for nitroaromatic detection in groundwater using living wild-type plants as selfpowered monitors. ${ }^{160}$ They embedded a pair of near-infrared fluorescent nanosensors into the plant leaf mesophyll. One nanosensor comprised single-walled carbon nanotubes (SWCNTs) conjugated to a peptide (Bombolitin II), which could recognize nitroaromatics via infrared fluorescent emission; another comprised polyvinyl-alcohol functionalized SWCNTs that acted as an invariant reference signal. The nitroaromatics in groundwater could be transported and accumulated in the mesophyll by the plant, which subsequently resulted in relative changes in the emission intensity. More interestingly, the infrared information could be sent to a smartphone, allowing easy real-time monitoring of the environment via infrared communication.

\section{Conclusions and perspectives}

Nanoscience/nanotechnology is currently one of the fastest developing fields. The mushrooming of new NMs greatly promotes advances being made in analytical science. In this article, we have reviewed recent progress in the application of NMs in the analysis of ECCs. It can be clearly recognized that NMs are taking more and more prominent roles in the development of analytical methods for ECCs. In a wide range of analytical techniques, NMs have shown their superior capabilities over conventional materials in enhancing the sensitivity and selectivity of detection, reducing the analytical time and sample consumption, and enabling miniaturization of the analytical devices. From the recent progress, we can elucidate that this field has shown the following clear trends:

(1) More NMs are finding roles in the analysis of ECCs. In the past, it usually took a long period of time to recognize the application potential of a new material in environmental analysis after it was synthesized and applied in other fields. This situation has now changed a lot because more and more analytical chemists have realized the great value of NMs in analytical chemistry.

(2) The designing and functionalization of NMs is becoming more elaborate. In most cases, multiple components or functional groups are integrated into the nanoadsorbents or nanoprobes to make the materials versatile in different aspects of analysis (e.g., separation, enrichment, detection).

(3) More modern analytical techniques (e.g., microextraction, microfluidics, nanoelectrodes, high-resolution and highthroughput mass spectrometry) are being used together with NMs to better fulfill the requirements for the analysis of ECCs.

(4) More stress is being placed on the analytical ability of complex samples. The methods are usually not only validated with artificial spiked samples, but also need to be applied in different types of real-world samples.
Undoubtedly, NMs now provide powerful analytical tools for the research on ECCs. Nevertheless, we should also be aware of the current challenges remaining in this field. Most of these new tools are still used only in the lab and cannot be immediately put into practical applications. To solve this problem, in future studies, more effort should be made in the following aspects:

(1) Standardization and commercialization of NMs. The reproducibility in the synthesis of NMs in different batches and from different labs is still a serious problem. Small differences in the properties of the NMs can lead to proportionally large deviations in the analytical results. Thus, there is an urgent need to push forward on the standardization of NMs to give more confidence to the analytical results obtained by NMsbased methods. It is also highly desirable that more NMs should be commercially available so that one can easily obtain NMs to use them in the analysis of ECCs.

(2) Reducing the cost of NMs. The synthesis and purification of NMs is still not an easy task in most environmental labs. However, compared with traditional materials, the high cost of commercial NMs greatly reduces their attractiveness. It is also very important to increase the reusability and recyclability of NMs to make them more cost-effective.

(3) More practical application-oriented method development. Despite the delicate designing of NMs, their performance in practical use is usually not as good as expected, mainly due to an insufficient consideration of environmental affecting factors (e.g., salinity, dissolved organic matter (DOM), $\mathrm{pH}$, temperature). Thus, the method development should be more practical application-oriented to fill the gap between method developers and method users regarding ECCs.

(4) More consideration of the requirements in modern environmental science. Elucidation of the relationship between environmental pollution and human health has become an important objective in modern environmental science. This raises a tremendous demand in analytical methods capable of in situ, in vivo, real-time, non-destructive, micro-volume, or high-throughput analysis of complex samples. NMs will definitely make a great contribution to achieve these goals.

\section{Acknowledgements}

The authors acknowledge financial support from the Chinese Academy of Sciences (No. XDB14010400, QYZDB-SSW-DQC018) and the National Natural Science Foundation of China (No. 21377141, 21422509, 21337002). S. Yao acknowledges the support from the National Natural Science Foundation of China (21235002, 21475038). Q. Liu also acknowledges the support from the Youth Innovation Promotion Association of CAS.

\section{References}

1 C. G. Daughton, Renew. Resour. J., 2005, 23, 6-23.

2 M. La Farre, S. Pérez, L. Kantiani and D. Barceló, Trends Anal. Chem., 2008, 27, 991-1007.

3 T. Deblonde, C. Cossu-Leguille and P. Hartemann, Int. J. Hyg. Environ. Health, 2011, 214, 442-448. 
4 Q. Liu, Q. Zhou and G. Jiang, Trends Anal. Chem., 2014, 58, 10-22.

5 D. Lapworth, N. Baran, M. Stuart and R. Ward, Environ. Pollut., 2012, 163, 287-303.

6 S. D. Richardson, Anal. Chem., 2010, 82, 4742-4774.

7 S. D. Richardson and T. A. Ternes, Anal. Chem., 2011, 83, 4614-4648.

8 S. A. Snyder, J. - Am. Water Works Assoc., 2014, 106, 38-52.

9 C. G. Daughton, J. Am. Soc. Mass Spectrom., 2001, 12, 10671076.

10 S. D. Richardson, Anal. Chem., 2011, 84, 747-778.

11 C. R. Martin, Nanomaterials: A Membrane-Based Synthetic Approach, DTIC Document, 1994.

12 G. Cao, Nanostructures and Nanomaterials: Synthesis, Properties and Applications, World Scientific, 2004.

13 J. A. Barreto, W. O'Malley, M. Kubeil, B. Graham, H. Stephan and L. Spiccia, Adv. Mater., 2011, 23, 18-40.

14 S. J. Klaine, P. J. Alvarez, G. E. Batley, T. F. Fernandes, R. D. Handy, D. Y. Lyon, S. Mahendra, M. J. McLaughlin and J. R. Lead, Environ. Toxicol. Chem., 2008, 27, 1825-1851.

15 Z. Wang, W. Zhu, Y. Qiu, X. Yi, A. von dem Bussche, A. Kane, H. Gao, K. Koski and R. Hurt, Chem. Soc. Rev., 2016, 45, 1750-1780.

16 Y. Wen, L. Chen, J. Li, D. Liu and L. Chen, Trends Anal. Chem., 2014, 59, 26-41.

17 S. C. Smith and D. F. Rodrigues, Carbon, 2015, 91, 122-143.

18 M. Govindhan, B.-R. Adhikari and A. Chen, RSC Adv., 2014, 4, 63741-63760.

19 S. Viswanathan and P. Manisankar, J. Nanosci. Nanotechnol., 2015, 15, 6914-6923.

20 J. Wang, Q. Liu, Y. Liang and G. Jiang, Anal. Bioanal. Chem., 2016, 408, 2861-2873.

21 J. Bian and S. V. Olesik, Analyst, 2017, 142, 1125-1132.

22 Y.-K. Kim, H.-K. Na, S.J. Kwack, S.-R. Ryoo, Y. Lee, S. Hong, S. Hong, Y. Jeong and D.-H. Min, ACS Nano, 2011, 5, 4550-4561.

23 T. Watanabe, H. Kawasaki, T. Yonezawa and R. Arakawa, J. Mass Spectrom., 2008, 43, 1063-1071.

24 C. Buzea, I. I. Pacheco and K. Robbie, Biointerphases, 2007, 2, MR17-MR71.

25 M. Farré, K. Gajda-Schrantz, L. Kantiani and D. Barceló, Anal. Bioanal. Chem., 2009, 393, 81-95.

26 S. Sharifi, S. Behzadi, S. Laurent, M. L. Forrest, P. Stroeve and M. Mahmoudi, Chem. Soc. Rev., 2012, 41, 2323-2343.

27 Q. Liu, M. Cheng, J. Wang and G. Jiang, Chem.- Eur. J., 2015, 21, 5594-5599.

28 Q. Liu, M. Cheng and G. Jiang, Chem.- Eur. J., 2013, 19, 5561-5565.

29 C. Zhang, Z. Zhang and G. Li, J. Chromatogr. A, 2014, 1346, 8-15.

30 K. Feng, B. Tang and P. Wu, J. Mater. Chem. A, 2014, 2, 16083-16092.

31 G. Zhao, L. Jiang, Y. He, J. Li, H. Dong, X. Wang and W. Hu, Adv. Mater., 2011, 23, 3959-3963.

32 X. Huang, Q. Liu, X. Huang, Z. Nie, T. Ruan, Y. Du and G. Jiang, Anal. Chem., 2017, 89, 1307-1314.
33 R. R. Nair, W. Ren, R. Jalil, I. Riaz, V. G. Kravets, L. Britnell, P. Blake, F. Schedin, A. S. Mayorov and S. Yuan, Small, 2010, 6, 2877-2884.

34 K.-J. Jeon, Z. Lee, E. Pollak, L. Moreschini, A. Bostwick, C.-M. Park, R. Mendelsberg, V. Radmilovic, R. Kostecki, T. J. Richardson and E. Rotenberg, ACS Nano, 2011, 5, 1042-1046.

35 A. Ivanovskii, Prog. Solid State Chem., 2013, 41, 1-19.

36 D. Malko, C. Neiss, F. Viñes and A. Görling, Phys. Rev. Lett., 2012, 108, 086804.

37 Y. Zhang, Q. Pei and C. Wang, Appl. Phys. Lett., 2012, 101, 081909.

38 A. Brown and S. Rundqvist, Acta Crystallogr., 1965, 19, 684685.

39 L. Li, Y. Yu, G. J. Ye, Q. Ge, X. Ou, H. Wu, D. Feng, X. H. Chen and Y. Zhang, Nat. Nanotechnol., 2014, 9, 372377.

40 F. Xia, H. Wang and Y. Jia, Nat. Commun., 2014, 5, 4458.

41 J. Wang, M. Cheng, Z. Zhang, L. Guo, Q. Liu and G. Jiang, Chem. Commun., 2015, 51, 4619-4622.

42 J. Wang, Q. Liu, Y. Gao, Y. Wang, L. Guo and G. Jiang, Anal. Chem., 2015, 87, 6931-6936.

43 X. Huang, Q. Liu, J. Fu, Z. Nie, K. Gao and G. Jiang, Anal. Chem., 2016, 88, 4107-4113.

44 M. Wang, H. Yuan, W. Deng, W. Bi and X. Yang, J. Chromatogr. A, 2015, 1412, 12-21.

45 J. Yang, L. Si, S. Cui and W. Bi, Microchim. Acta, 2015, 182, 737-744.

46 S. Yang, Y. Gong, J. Zhang, L. Zhan, L. Ma, Z. Fang, R. Vajtai, X. Wang and P. M. Ajayan, Adv. Mater., 2013, 25, 2452-2456.

47 M. Rong, L. Lin, X. Song, T. Zhao, Y. Zhong, J. Yan, Y. Wang and X. Chen, Anal. Chem., 2014, 87, 1288-1296.

48 J. Schuster, G. He, B. Mandlmeier, T. Yim, K. T. Lee, T. Bein and L. F. Nazar, Angew. Chem., 2012, 124, 3651-3655.

$49 \mathrm{H}$. Zhou, S. Zhu, M. Hibino, I. Honma and M. Ichihara, $A d v$. Mater., 2003, 15, 2107-2111.

50 H.-B. Zheng, J.-Z. Mo, Y. Zhang, Q. Gao, J. Ding, Q.-W. Yu and Y.-Q. Feng, J. Chromatogr. A, 2014, 1329, 17-23.

51 L. Zhou, L. Ji, P.-C. Ma, Y. Shao, H. Zhang, W. Gao and Y. Li, J. Hazard. Mater., 2014, 265, 104-114.

52 Z. Y. Gu, C. X. Yang, N. Chang and X. P. Yan, Acc. Chem. Res., 2012, 45, 734-745.

53 H. Li, M. Eddaoudi, M. O'Keeffe and O. M. Yaghi, Nature, 1999, 402, 276-279.

54 O. K. Farha and J. T. Hupp, Acc. Chem. Res., 2010, 43, 11661175.

55 J. J. Perry Iv, J. A. Perman and M. J. Zaworotko, Chem. Soc. Rev., 2009, 38, 1400-1417.

56 N. A. Khan, Z. Hasan and S. H. Jhung, J. Hazard. Mater., 2013, 244, 444-456.

57 K. A. Cychosz and A. J. Matzger, Langmuir, 2010, 26, 1719817202.

58 X. Feng, X. S. Ding and D. L. Jiang, Chem. Soc. Rev., 2012, 41, 6010-6022.

59 S. Y. Ding and W. Wang, Chem. Soc. Rev., 2013, 42, 548-568. 
60 A. P. Cote, A. I. Benin, N. W. Ockwig, M. O'Keeffe, A. J. Matzger and O. M. Yaghi, Science, 2005, 310, 11661170.

61 J. Chen and J. X. Zhao, Sensors, 2012, 12, 2414-2435.

62 X. Liu, C.-H. Yan and J. A. Capobianco, Chem. Soc. Rev., 2015, 44, 1299-1301.

63 D. Yang, Z. Hou, Z. Cheng, C. Li and J. Lin, Chem. Soc. Rev., 2015, 44, 1416-1448.

64 S. Zhu, Q. Meng, L. Wang, J. Zhang, Y. Song, H. Jin, K. Zhang, H. Sun, H. Wang and B. Yang, Angew. Chem., Int. Ed., 2013, 52, 3953-3957.

65 Y.-P. Sun, B. Zhou, Y. Lin, W. Wang, K. S. Fernando, P. Pathak, M. J. Meziani, B. A. Harruff, X. Wang, H. Wang, P. G. Luo, H. Yang, M. E. Kose, B. Chen, L. M. Veca and S.-Y. Xie, J. Am. Chem. Soc., 2006, 128, 7756-7757.

66 R. Liu, D. Wu, S. Liu, K. Koynov, W. Knoll and Q. Li, Angew. Chem., 2009, 121, 4668-4671.

67 S. Y. Lim, W. Shen and Z. Gao, Chem. Soc. Rev., 2015, 44, 362-381.

68 J. Hou, H. Li, L. Wang, P. Zhang, T. Zhou, H. Ding and L. Ding, Talanta, 2016, 146, 34-40.

69 P. D. Cozzoli, A. Kornowski and H. Weller, J. Am. Chem. Soc., 2003, 125, 14539-14548.

70 S. M. Gupta and M. Tripathi, Cent. Eur. J. Chem., 2012, 10, 279-294.

71 C. Shanshan, Z. Yinhua, L. Wei, L. Weijia, L. Licheng, Y. Zhuhong, L. Chang, Y. Wenjun, L. Xiaohua and F. Xin, Chin. J. Catal., 2010, 31, 605-614.

72 A. González, J. Avivar, F. Maya, C. P. Cabello, G. T. Palomino and V. Cerdà, Anal. Bioanal. Chem., 2017, 409, 225-234.

73 T. Wang, J. Wang, C. Zhang, Z. Yang, X. Dai, M. Cheng and X. Hou, Analyst, 2015, 140, 5308-5316.

74 I. Velzeboer, C. Kwadijk and A. Koelmans, Environ. Sci. Technol., 2014, 48, 4869-4876.

75 B. Beless, H. S. Rifai and D. F. Rodrigues, Environ. Sci. Technol., 2014, 48, 10372-10379.

76 A. Speltini, M. Sturini, F. Maraschi, L. Consoli, A. Zeffiro and A. Profumo, J. Chromatogr. A, 2015, 1379, 9-15.

77 S.-D. Pan, L.-X. Zhou, Y.-G. Zhao, X.-H. Chen, H.-Y. Shen, M.-Q. Cai and M.-C. Jin, J. Chromatogr. A, 2014, 1362, 34-42.

78 Y. Patiño, E. Díaz, S. Ordóñez, E. Gallegos-Suarez, A. Guerrero-Ruiz and I. Rodríguez-Ramos, Chemosphere, 2015, 136, 174-180.

79 M. L. Chen, J. Q. Min, M. Q. Cai, S. D. Pan and M. C. Jin, J. Sep. Sci., 2016, 39, 2396-2405.

80 J. Zhou, T. Han, H. Ma, T. Yan, X. Pang, Y. Li and Q. Wei, Anal. Chim. Acta, 2015, 889, 82-89.

81 M. A. Ali, K. K. Reza, S. Srivastava, V. V. Agrawal, R. John and B. D. Malhotra, Langmuir, 2014, 30, 4192-4201.

82 C.-X. Xu, Z.-W. Wu, F.-Z. Cao and Y.-Y. Gao, Metall. Anal., 2010, 30, 30-34.

83 X. Zhou, Y. Zhang, Z. Huang, D. Lu, A. Zhu and G. Shi, Sci. Rep., 2016, 6, 38417.

84 Y. Zhuang, F. Yu and J. Ma, J. Nanomater., 2015, 2015, 8.

85 Y. Wang, W. Wang, H. Mao, Y. Lu, J. Lu, J. Huang, Z. Ye and B. Lu, ACS Appl. Mater. Interfaces, 2014, 6, 12698-12706.
86 S. C. Smith, F. Ahmed, K. M. Gutierrez and D. F. Rodrigues, Chem. Eng. J., 2014, 240, 147-154.

87 T. Lammel, P. Boisseaux and J. M. Navas, Environ. Toxicol., 2015, 30, 1192-1204.

88 J. Wang, Z. Chen and B. Chen, Environ. Sci. Technol., 2014, 48, 4817-4825.

89 X. Chen and B. Chen, Environ. Sci. Technol., 2015, 49, 61816189.

90 A. Speltini, M. Maiocchi, L. Cucca, D. Merli and A. Profumo, Anal. Bioanal. Chem., 2014, 406, 3657-3665.

91 J. Comer, R. Chen, H. Poblete, A. Vergara-Jaque and J. E. Riviere, ACS Nano, 2015, 9, 11761-11774.

92 N. Cai and P. Larese-Casanova, J. Colloid Interface Sci., 2014, 426, 152-161.

93 F. Wang, J. J.-H. Haftka, T. L. Sinnige, J. L. Hermens and W. Chen, Environ. Pollut., 2014, 186, 226-233.

94 H. Guo, T. Jiao, Q. Zhang, W. Guo, Q. Peng and X. Yan, Nanoscale Res. Lett., 2015, 10, 272.

95 L. Wang, L. Jiang, D. Su, C. Sun, M. Chen, K. Goh and Y. Chen, J. Colloid Interface Sci., 2014, 430, 121-128.

96 L. A. Al-Khateeb, S. Almotiry and M. A. Salam, Chem. Eng. J., 2014, 248, 191-199.

97 N. Sun, Y. Han, H. Yan and Y. Song, Anal. Chim. Acta, 2014, 810, 25-31.

98 H. Chen, B. Gao and H. Li, J. Hazard. Mater., 2015, 282, 201207.

99 H. B. Shang, C. X. Yang and X. P. Yan, J. Chromatogr. A, 2014, 1357, 165-171.

100 X. Liu, T. Feng, C. Wang, L. Hao, C. Wang, Q. Wu and Z. Wang, Anal. Methods, 2016, 8, 3528-3535.

101 X. Dai, X. Jia, P. Zhao, T. Wang, J. Wang, P. Huang, L. He and X. Hou, Talanta, 2016, 154, 581-588.

102 S. Lirio, W.-L. Liu, C.-L. Lin, C.-H. Lin and H.-Y. Huang, J. Chromatogr. A, 2016, 1428, 236-245.

103 Y. Li, C.-X. Yang and X.-P. Yan, Chem. Commun., 2017, 53, 2511-2514.

104 N. Wang, L. Zhou, J. Guo, Q. Ye, J.-M. Lin and J. Yuan, Appl. Surf. Sci., 2014, 305, 267-273.

105 X. Zang, Q. Chang, M. Hou, C. Wang and Z. Wang, Anal. Methods, 2015, 7, 8793-8800.

106 A. Mehdinia, N. Khodaee and A. Jabbari, Anal. Chim. Acta, 2015, 868, 1-9.

107 D. Huang, X. Wang, C. Deng, G. Song, H. Cheng and X. Zhang, J. Chromatogr. A, 2014, 1325, 65-71.

108 X. Cao, L. Shen, X. Ye, F. Zhang, J. Chen and W. Mo, Analyst, 2014, 139, 1938-1944.

109 X. Wang and C. Deng, Talanta, 2015, 144, 1329-1335.

110 N. Gan, J. Zhang, S. Lin, N. Long, T. Li and Y. Cao, Materials, 2014, 7, 6028-6044.

111 S. Chinthakindi, A. Purohit, V. Singh, V. Tak, D. Dubey and D. Pardasani, J. Chromatogr. A, 2015, 1423, 54-62.

112 L. Li, R. Guo, Y. Li, M. Guo, X. Wang and X. Du, Anal. Chim. Acta, 2015, 867, 38-46.

113 F. Brandl, N. Bertrand, E. M. Lima and R. Langer, Nat. Commun., 2015, 6, 7765.

114 P. Singla, N. Goel and S. Singhal, Chem. Eng. J., 2016, 299, 403-414. 
115 A. Schiwy, H. M. Maes, D. Koske, M. Flecken, K. R. Schmidt, H. Schell, A. Tiehm, A. Kamptner, S. Thümmler and H. Stanjek, Environ. Pollut., 2016, 216, 419-427.

116 S. B. Khan, A. M. Asiri, K. Akhtar and M. A. Rub, J. Ind. Eng. Chem., 2015, 30, 234-238.

117 F. Zhou, Y. Wang, W. Wu, T. Jing, S. Mei and Y. Zhou, Sci. Rep., 2016, 6, 38000.

118 A. Pal, M. P. Sk and A. Chattopadhyay, ACS Appl. Mater. Interfaces, 2016, 8, 5758-5762.

119 A. Nguyen, C. M. Gonzalez, R. Sinelnikov, W. Newman, S. Sun, R. Lockwood, J. G. Veinot and A. Meldrum, Nanotechnology, 2016, 27, 105501.

120 S. Benítez-Martínez, A. N. I. López-Lorente and M. Valcárcel, Anal. Chem., 2014, 86, 12279-12284.

121 G. Hu, W. Sheng, Y. Zhang, X. Wu and S. Wang, Anal. Bioanal. Chem., 2015, 407, 8487-8496.

122 G. Hu, W. Sheng, Y. Zhang, J. Wang, X. Wu and S. Wang, J. Agric. Food Chem., 2016, 64, 3908-3915.

123 C. Liu, Z. Song, J. Pan, Y. Yan, Z. Cao, X. Wei, L. Gao, J. Wang, J. Dai and M. Meng, Talanta, 2014, 125, 14-23.

124 N. Duan, H. Zhang, Y. Nie, S. Wu, T. Miao, J. Chen and Z. Wang, Anal. Methods, 2015, 7, 5186-5192.

125 A. Sharma, A. Hayat, R. K. Mishra, G. Catanante, S. Bhand and J. L. Marty, Toxins, 2015, 7, 3771-3784.

126 X. Deng, X. Huang and D. Wu, Anal. Bioanal. Chem., 2015, 407, 4607-4613.

127 H. Huang, Z. Feng, Y. Li, Z. Liu, L. Zhang, Y. Ma and J. Tong, Anal. Methods, 2015, 7, 2928-2935.

128 M. Kalita, S. Balivada, V. P. Swarup, C. Mencio, K. Raman, U. R. Desai, D. Troyer and B. Kuberan, J. Am. Chem. Soc., 2014, 136, 554-557.

129 Z. Tan, H. Xu, G. Li, X. Yang and M. M. Choi, Spectrochim. Acta, Part A, 2015, 149, 615-620.

130 H.-L. Meng, G.-H. Chen, X. Guo, P. Chen, Q.-H. Cai and Y.-F. Tian, Anal. Bioanal. Chem., 2014, 406, 3201-3208.

131 Y.-B. Miao, H.-X. Ren, N. Gan, Y. Zhou, Y. Cao, T. Li and Y. Chen, Anal. Chim. Acta, 2016, 929, 49-55.

132 M. Ma, K. Wen, R. C. Beier, S. A. Eremin, C. Li, S. Zhang, J. Shen and Z. Wang, ACS Appl. Mater. Interfaces, 2016, 8, 17745-17750.

133 J. V. Rohit, R. K. Singhal and S. K. Kailasa, Sens. Actuators, B, 2016, 237, 1044-1055.

134 D. Wen, W. Liu, A. K. Herrmann, D. Haubold, M. Holzschuh, F. Simon and A. Eychmüller, Small, 2016, 12, 2439-2442.

135 Y. Tian, Y. Wang, Z. Sheng, T. Li and X. Li, Anal. Biochem., 2016, 513, 87-92.

136 K. M. Kim, Y.-S. Nam, I. W. Nah, Y. Lee and K.-B. Lee, J. Nanosci. Nanotechnol., 2016, 16, 11191-11200.

137 S. Wu, N. Duan, W. Zhang, S. Zhao and Z. Wang, Anal. Biochem., 2016, 508, 58-64.

138 V. Kumar, S. Mohan, D. K. Singh, D. K. Verma, V. K. Singh and S. H. Hasan, Mater. Sci. Eng., C, 2017, 71, 1004-1019.

139 F. Yuan, H. Zhao, H. Zang, F. Ye and X. Quan, ACS Appl. Mater. Interfaces, 2016, 8, 9855-9864.

140 J. R. Bhamore, P. Ganguly and S. K. Kailasa, Sens. Actuators, $B, 2016,233,486-495$.
141 F. Qu, P. Chen, S. Zhu and J. You, Spectrochim. Acta, Part A, 2017, 171, 449-453.

142 L. Yang, X. Liu, Q. Lu, N. Huang, M. Liu, Y. Zhang and S. Yao, Anal. Chim. Acta, 2016, 930, 23-30.

143 C. N. Iswarya, S. K. Daniel and M. Sivakumar, Mater. Sci. Eng., C, 2017, 75, 393-401.

144 M. Amjadi, T. Hallaj, H. Asadollahi, Z. Song, M. de Frutos and N. Hildebrandt, Sens. Actuators, B, 2017, 244, 425-432.

145 R. Alam, I. V. Lightcap, C. J. Karwacki and P. V. Kamat, ACS Nano, 2014, 8, 7272-7278.

146 J. Chen, S. Feng, F. Gao, E. Grant, J. Xu, S. Wang, Q. Huang and X. Lu, J. Food Sci., 2015, 80, N834-N840.

147 Y. Zhang, Z. Wang, L. Wu, Y. Pei, P. Chen and Y. Cui, Analyst, 2014, 139, 5148-5154.

148 T. Yang, Z. Zhang, B. Zhao, R. Hou, A. Kinchla, J. M. Clark and L. He, Anal. Chem., 2016, 88, 5243-5250.

149 S. Yang, X. Dai, B. B. Stogin and T.-S. Wong, Proc. Natl. Acad. Sci. U. S. A., 2016, 113, 268-273.

150 Q. Ma, H. Zhang, W. Liu, J. Ge, J. Wu, S. Wang and P. Wang, RSC Adv., 2016, 6, 85285-85292.

151 M. Qi, X. Huang, Y. Zhou, L. Zhang, Y. Jin, Y. Peng, H. Jiang and S. Du, Food Chem., 2016, 197, 723-729.

152 M. Merchant and S. R. Weinberger, Electrophoresis, 2000, 21, 1164-1177.

153 R. E. Caffrey, in The Urinary Proteome: Methods and Protocols, Springer, 2010, pp. 167-183.

154 W. E. Grizzle, O. J. Semmes, W. Bigbee, L. Zhu, G. Malik, D. K. Oelschlager, B. Manne and U. Manne, Cancer Inf., 2005, 1, 86-97.

155 Q. Min, X. Zhang, X. Chen, S. Li and J.-J. Zhu, Anal. Chem., 2014, 86, 9122-9130.

156 Z. Rao, F. Geng, Y. Zhou, D. Cao and Y. Kang, Anal. Methods, 2017, 9, 2014-2020.

157 H.-L. Liu, Y.-J. Chang, T. Fan and Z.-Y. Gu, Chem. Commun., 2016, 52, 12984-12987.

158 Z. Lin, J. Zheng, G. Lin, Z. Tang, X. Yang and Z. Cai, Anal. Chem., 2015, 87, 8005-8012.

159 W. Li, P. Sheng, J. Cai, H. Feng and Q. Cai, Biosens. Bioelectron., 2014, 61, 209-214.

160 M. H. Wong, J. P. Giraldo, S.-Y. Kwak, V. B. Koman, R. Sinclair, T. T. S. Lew, G. Bisker, P. Liu and M. S. Strano, Nat. Mater., 2017, 16, 264-272.

161 E. Khaled, M. S. Kamel, H. Hassan, H. Abdel-Gawad and H. Y. Aboul-Enein, Talanta, 2014, 119, 467-472.

162 X. Lu, X. Wang, J. Jin, Q. Zhang and J. Chen, Biosens. Bioelectron., 2014, 62, 134-139.

163 J. Li, J. Li, H. Feng, Y. Zhang, J. Jiang, Y. Feng, M. Chen and D. Qian, Electrochim. Acta, 2015, 153, 352-360.

164 R. J. Rubira, S. A. Camacho, P. H. Aoki, F. V. Paulovich, O. N. Oliveira and C. J. Constantino, J. Mater. Sci., 2016, 51, 3182-3190.

165 H. Luo, Y. Huang, K. Lai, B. A. Rasco and Y. Fan, Food Contr., 2016, 68, 229-235.

166 J. Chen, Y.-W. Huang and Y. Zhao, J. Mater. Chem. B, 2015, 3, 1898-1906. 